This item was submitted to Loughborough's Research Repository by the author.

Items in Figshare are protected by copyright, with all rights reserved, unless otherwise indicated.

\title{
Closed-form stochastic response of linear building structures to spectrum- consistent seismic excitations
}

PLEASE CITE THE PUBLISHED VERSION

\section{PUBLISHER}

Elsevier

\section{VERSION}

AM (Accepted Manuscript)

\section{PUBLISHER STATEMENT}

This paper was accepted for publication in the journal Soil Dynamics and Earthquake Engineering and the definitive published version is available at https://doi.org/10.1016/j.soildyn.2019.105724.

\section{LICENCE}

CC BY-NC-ND 4.0

\section{REPOSITORY RECORD}

Barone, Giorgio, Francesco Lo lacono, Giacomo Navarra, and Alessandro Palmeri. 2019. "Closed-form Stochastic Response of Linear Building Structures to Spectrum-consistent Seismic Excitations". figshare. https://hdl.handle.net/2134/9642596.v1. 


\title{
Closed-form stochastic response of linear building structures to spectrum-consistent seismic excitations
}

\author{
Giorgio Barone ${ }^{\mathrm{a}}$, Francesco Lo Iacono ${ }^{\mathrm{b}}$, Giacomo Navarra ${ }^{\mathrm{b}, *}$, Alessandro Palmeri ${ }^{\mathrm{a}}$ \\ ${ }^{a}$ School of Architecture, Building and Civil Engineering, Loughborough University, Loughborough, Leicestershire LE11 3TU, United Kingdom. \\ ${ }^{b}$ Facoltà di Ingegneria ed Architettura, Università degli Studi di Enna "Kore", Cittadella Universitaria, Enna Bassa I-94100 Enna, Italy.
}

\begin{abstract}
Design codes typically define the seismic action in terms of pseudo-acceleration response spectra, encouraging the use of the modal superposition method for the evaluation of the structural response. For linear structural systems, provided that the ground shaking has been appropriately modelled, the full characterization of the response processes of interest can be achieved with the application of the random vibration theory. An analytical model for the power spectral density (PSD) functions consistent with seismic response spectra has been recently proposed. In this paper, taking advantage of this novel PSD model, closed-form approximate expressions of the spectral moments of the structural response are derived and numerically validated for single- and multi-degree of freedom systems. The proposed formulation is applied to the case of base isolated multi-story buildings, aimed at overcoming the difficulties associated with the non-classical nature of their damping. The paper shows how the proposed approach can be used for an accurate and computationally efficient evaluation of the probabilistic distribution of the structural response maxima.
\end{abstract}

Keywords: Base-isolated building, First-passage problem, Response spectrum, Spectrum-consistent PSD, Stochastic analysis.

\section{Introduction}

2 The seismic response of a structural system depends on both its dynamical properties and the characteristics of the ${ }_{3}$ ground motion time histories. Indeed, the evaluation of the response is strongly dependent on the mathematical model ${ }_{4}$ chosen to describe the seismic input and often needs to be carried out in a probabilistic framework. In several design 5 scenarios, earthquakes can be modelled as stationary zero-mean Gaussian processes [1]. In this case, the seismic input ${ }_{6}$ can be probabilistically defined by its power spectral density (PSD) function [2]. The latter carries several advantages: 7 for linear systems, a full characterization of the response process can be easily achieved by means of spectral analysis; 8 for nonlinear systems, it enables the artificial generation of ground motion time-history samples, matching the design $\vartheta$ response spectra (RS), in order to perform Monte-Carlo simulations (MCS).

${ }_{10}$ In the last decades, great attention has been devoted to the development of analytical and numerical models for ${ }_{11}$ RS-consistent PSD functions. Earlier contributions can be found in the review paper by Ahmadi [3]. A common 12 approach to model earthquake time histories is to use time-varying envelope functions that modulates realizations of is a stationary PSD expressed either by Tajimi-Kanai $[4,5]$ or Clough-Penzien [1] models. As an example, Falsone 14 et al. [6] developed a technique to obtain the filter coefficients for the design RS of Eurocode 8 [7]. Several other 15 approaches have been proposed to numerically generate PSD functions consistent with non-smooth RS (e.g. for the ${ }_{16}$ design of special structures, such as nuclear power plants). However, these techniques generally require numerical 17 iterative procedures, either in the time or in the frequency domain [8-15]. Further methods have been developed to 18 obtain sets of RS-consistent acceleration time histories, which are also able to mimic the non-stationary characteristics 19 of field-recorded accelerograms [16-25] by means of the definition of evolutionary PSD functions [26-28].

\footnotetext{
${ }^{*}$ Corresponding author
} 
Recently, a novel formulation for the PSD function consistent with the design RS of Eurocode 8 and the former ${ }_{21}$ Italian National Code has been proposed [29-31], using an analytical model, whose parameters are expressed as 22 functions of the target RS parameters [32].

For linear structural systems, once the PSD function of the input ground motion is known, stochastic analysis ${ }_{24}$ allows determining the PSD function of the structural response. Then, various indicators of the structural safety ${ }_{25}$ can be computed by using the spectral moments (SMs) of the dynamic respose [33-35]. Closed-form solutions ${ }_{26}$ of SMs have been determined only in some particular cases [36, 37]. In general, they are evaluated by means of ${ }_{27}$ numerical integration, resulting in a significant computational effort, especially for large multi-degree-of-freedom 28 (MDOF) systems [38].

Furthermore, RS-consistent PSD functions have been recently used also for non-linear stochastic dynamic analso yses of yielding structures through the application of the statistical linearisation technique $[39,40]$. In the case of ${ }_{31}$ multi-storey inelastic building structures, the approach in Ref. [41] has been proposed to estimate the response peak ${ }_{32}$ values without the integration of the non-linear equations of motion and operating directly on the physical domain. ${ }_{3}$ In this way, no further approximations related to modal decomposition are needed. Coupling such technique with an ${ }_{34}$ analytical model of the PSD function of the seismic input could reduce the overall computational effort.

35 In this paper, exploiting the analytical PSD model proposed in Ref. [32], the authors propose novel closed-form ${ }_{36}$ expressions for the SMs of linear structural systems excited by RS-consistent seismic input. SMs are first derived as ${ }_{37}$ functions of the parameters defining both the SDOF (single-degree-of-freedom) oscillators and the RS; then, direct ${ }_{38}$ and cross SMs of order up to two are obtained in closed-form for MDOF systems. Finally, these expressions are з9 applied to the case of multi-story base-isolated buildings by applying a convenient double modal transformation of ${ }_{40}$ coordinates [42]. The proposed formulae are validated against MCS. The authors also show how using the analyti${ }_{41}$ cal SMs allows an accurate reproduction of the target RS, with reference to Eurocode 8 [7] and other international 42 codes, including 2-, 3- and 4-branch design RS. Finally, the paper shows the significant advantage of SMs closed${ }_{43}$ form expressiond for the probabilistic assessment of the structural response by analytically computing the cumulative ${ }_{44}$ distribution functions (CDFs) of the response maxima. In the latter case, the authors show how the proposed method 45 achieves improved accuracy and significantly reduces the computational effort.

\section{${ }_{46}$ 2. Power Spectral Density functions consistent to Response Spectra}

${ }_{47} \mathrm{RS}$ are widely used in earthquake engineering to represent the peak values of the dynamic response (e.g. in terms 48 of displacements, velocities, accelerations) of linear and nonlinear SDOF oscillators to a specified ground motion as 49 a function of its natural period of vibration and its damping capabilities [43]. For instance, assuming linear viscous ${ }_{50}$ damping, the RS in terms of displacements can be expressed as:

$$
S_{\mathrm{d}}(T, \zeta)=\max \{|u(t)|\},
$$

${ }_{51}$ in which $T$ is the natural period of vibration, $\zeta$ is the viscous damping ratio and $u(t)$ is the time history of the response ${ }_{52}$ displacement.

${ }_{53}$ Most building codes define the design value of the seismic action at a given site by means of the expected pseudo${ }_{54}$ acceleration RS, depending on the seismicity of the site, the soil conditions, the importance of the structure, the ${ }_{55}$ assumed ductility of the structure and the limit state under consideration. This approach is strictly valid only for ${ }_{56} \mathrm{SDOF}$ systems, as the evaluation of the response for MDOF structures implies approximations in the determination ${ }_{57}$ of the modal response correlation coefficients [44, 45]. Despite this limitation, using the RS to predict the peak values ${ }_{58}$ of structural displacements and internal forces has the significant advantage to avoid the direct integration of the ${ }_{59}$ equations of motion.

60 Based on the current international seismic codes, a general class of pseudo-acceleration design RS can be described 


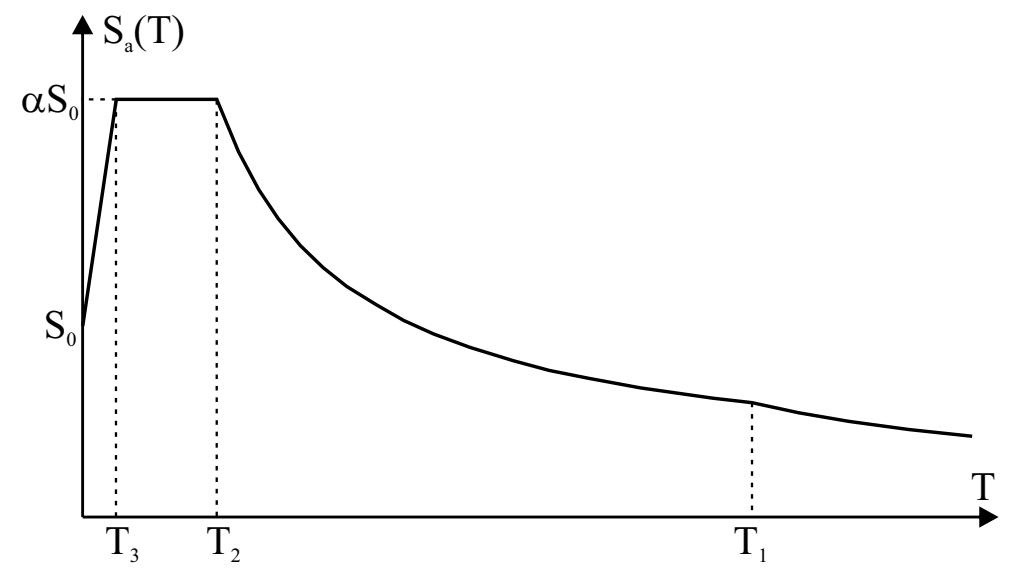

Figure 1: Response spectrum in terms of pseudo-accelerations.

61 using the following piecewise function:

$$
S_{\mathrm{pa}}(T)= \begin{cases}S_{0}\left[1+(\alpha-1) \frac{T}{T_{3}}\right], & 0 \leq T<T_{3} ; \\ \alpha S_{0}, & T_{3} \leq T \leq T_{2} ; \\ \alpha S_{0}\left(\frac{T_{2}}{T}\right)^{k_{1}}, & T_{2}<T \leq T_{1} ; \\ \alpha S_{0}\left(\frac{T_{2}}{T_{1}}\right)^{k_{1}}\left(\frac{T_{1}}{T}\right)^{k_{2}}, & T>T_{1},\end{cases}
$$

62 where $S_{0}$ is the peak ground acceleration (PGA), $\alpha$ is a dynamic amplification factor, $T_{1}>T_{2}>T_{3}$ are periods delim${ }_{63}$ iting the various RS branches, and $k_{1}$ and $k_{2}$ are shape factors. In the following, the authors explicitly refer to design ${ }_{64} \mathrm{RS}$ having $k_{1}=1$ and $k_{2}=2$ (Figure 1), so that the second, third and fourth branches correspond to constant spectral 65 pseudo-accelerations, pseudo-velocities and displacements, respectively, while the first branch linearly connects the ${ }_{66}$ point at $T=0$, i.e. the PGA to the second branch. Several major seismic codes (e.g., see Table 1) adopt similar RS 67 models, some only using three or two branches, that can be derived as special cases of Eq. (2).

68 Advanced seismic codes allow using alternative representations of seismic actions in terms of recorded, artificial 69 or simulated accelerograms. The latter can be generated as samples of a zero-mean Gaussian stationary process, 70 provided that the RS computed from the ensemble of the artificial accelerograms closely matches the design RS. An 71 advantage of this approach is that the PSD function of this process is related only to the ground acceleration, being 72 independent of the dynamic characteristics of the superimposed structure (e.g. viscous damping ratio, nonlinearities, 73 etc.).

\section{2.1. Numerical approach}

75 While evaluating a RS for a given PSD function is straightforward, the inverse problem (i.e. determining the PSD 76 function consistent to an assigned RS) is not. An estimate of the one-sided PSD function $G_{\ddot{U}_{\mathrm{g}}}(\omega)$ consistent with an 77 assigned RS can be obtained by considering the following implicit equation [14]:

$$
G_{\ddot{U}_{\mathrm{g}}}(\omega)=\frac{\gamma}{\omega}\left[\left(\frac{S_{\mathrm{pa}}(\omega, \zeta)}{\eta_{U}(\omega, \zeta)}\right)^{2}-\int_{0}^{\omega} G_{\ddot{U}_{\mathrm{g}}}(\hat{\omega}) \mathrm{d} \hat{\omega}\right],
$$

78 where $\gamma=4 \zeta /(\pi-4 \zeta), S_{\mathrm{pa}}(\omega, \zeta)$ is the target pseudo-acceleration RS and $\eta_{U}(\omega, \zeta)$ is the so-called Vanmarcke's peak ${ }_{79}$ factor for the displacement response process $U(t)[34]$. 
Table 1: Classification of seismic codes with design RS that can be expressed by Eq. (2) $\left(k_{1}=1\right.$ and $\left.k_{2}=2\right)$.

\begin{tabular}{llc}
\hline Country & Seismic Code & Year \\
\hline \multicolumn{3}{c}{ Four-branch design RS } \\
\hline Australia & AS1170.4 & 2007 \\
Colombia & NRS10 & 2010 \\
European Union & Eurocode 8 [7] & 2004 \\
\hline \multicolumn{3}{c}{ Three-branch design RS } \\
\hline Dominican Republic & Decreto 201/11 MOPC \\
India & Indian Standard 1893 & 2011 \\
Indonesia & SNI-1726-2002 \\
Japan & Building Standard Law & 2002 \\
Korea & Korea Building Code \\
Philippines & NSCP & 2002 \\
Taiwan & SDCB & 2005 \\
USA & International Building Code [46] & 2010 \\
\hline \multicolumn{3}{c}{2015} \\
\hline Albania & Two-branch design RS \\
Ecuador & KTP-N.2-89 \\
Macedonia & INEN-5 & 1989 \\
Peru & Tech. Reg. Code & 2001 \\
Serbia & E.030 [47] & 1990 \\
Uganda & Tech. Reg. Code & 2003 \\
\hline
\end{tabular}

80 An approximate evaluation of $G_{\ddot{U}_{g}}(\omega)$ can be determined by assuming the PSD function to be piecewise constant 81 at all arbitrary selected frequency intervals $\Delta \omega$, and replacing the integral in Eq. (3) with a summation:

$$
G_{\ddot{U}_{\mathrm{g}}}\left(\omega_{i}\right)=\frac{4 \zeta}{\pi \omega_{i}-4 \zeta \omega_{i-1}}\left[\left(\frac{S_{\mathrm{pa}}\left(\omega_{i}, \zeta\right)}{\eta_{U}\left(\omega_{i}, \zeta\right)}\right)^{2}-\Delta \omega \sum_{j=1}^{i-1} G_{\ddot{U}_{\mathrm{g}}}\left(\omega_{j}\right)\right] .
$$

82 Exploiting Eq. (4), Cacciola et al. [14] proposed a numerical procedure to estimate the PSD function consistent ${ }_{83}$ with an assigned RS. This technique proved to be effective also for non-smooth RS obtained from natural earthquake ${ }_{84}$ ground motion time histories [16], and its accuracy could be further improved by using an iterative corrective method, 85 as demonstrated in [39]. However, the recursive nature of this numerical method entails the entire process must be ${ }_{86}$ repeated every time a new RS is selected. An alternative iterative corrective method to best-fit, in the mean sense, a ${ }_{87}$ PSD function to a given response spectrum, offering improved accuracy, has been proposed in Ref. [41].

\section{2.2. Analytical model}

${ }_{89}$ As part of the work reported in Ref. [32], an extensive numerical campaign has been executed, aimed at calculating 90 and comparing the spectrum-consistent PSD functions which satisfy Eq. (4) for assigned RS belonging to the class ${ }_{91}$ defined in Eq. (2). That is, a large number of spectrum-consistent PSD functions have been obtained numerically by 92 adopting the method proposed in Ref. [14], varying the parameters $\alpha, S_{0}, T_{1}, T_{2}$ and $T_{3}$ into Eq. (2). Specifically, ${ }_{93}$ the parameters have been varied in the following intervals: $\alpha \in[2.0,3.5] ; T_{3} / \mathrm{s} \in[0.05,0.20] ; T_{2} / \mathrm{s} \in[0.20,0.60]$; ${ }_{94} T_{1} / \mathrm{s} \in[1.50,3.50]$; while $S_{0}$ has been kept equal to 1 , as its value does not affect the shape of RS and PSD function, 95 and the duration $T_{\mathrm{S}}$ of the stationary part of the ground shaking, affecting the parameter $\eta_{U}$ in Eqs. (3) and (4), has ${ }_{96}$ been varied between 10 and $30 \mathrm{~s}$. The analyses have been repeated for 2-, 3- and 4-branch RS, covering all the building ${ }_{97}$ codes listed in Table 1. It has been observed that, for all these cases, Eq. (4) returns PSD functions having the shape ${ }_{98}$ qualitatively depicted in Figure 2, irrespectively of the soil type, i.e. from very stiff to very soft; this has also been 


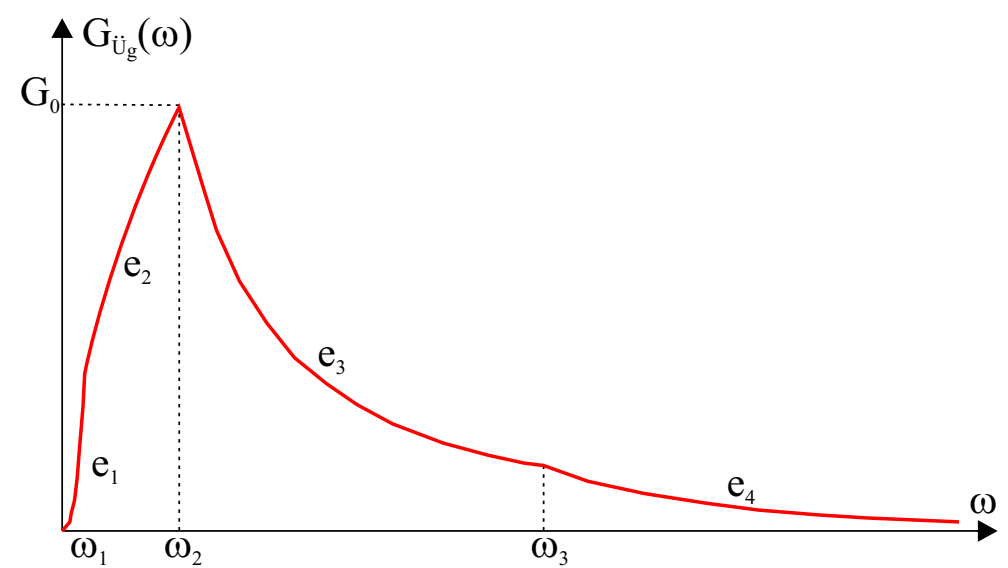

Figure 2: Power Spectral Density function consistent with Response Spectrum.

99 shown by further studies (see, among others, Refs. [39-41]). Importantly, the numerical investigations have proved 100 that each branch of the spectrum-consistent PSD function can be analytically fitted by an exponential law. This in turn 101 led to the development of the analytical model defined by the following piecewise expression:

$$
G_{\ddot{U}_{\mathrm{g}}}(\omega)= \begin{cases}G_{0}\left(\frac{\omega_{1}}{\omega_{2}}\right)^{e_{2}}\left(\frac{\omega}{\omega_{1}}\right)^{e_{1}}, & 0 \leq \omega<\omega_{1} ; \\ G_{0}\left(\frac{\omega}{\omega_{2}}\right)^{e_{2}}, & \omega_{1} \leq \omega<\omega_{2} ; \\ G_{0}\left(\frac{\omega}{\omega_{2}}\right)^{e_{3}}, & \omega_{2} \leq \omega \leq \omega_{3} ; \\ G_{0}\left(\frac{\omega_{3}}{\omega_{2}}\right)^{e_{3}}\left(\frac{\omega}{\omega_{3}}\right)^{e_{4}}, & \omega>\omega_{3},\end{cases}
$$

102 where $G_{0}$ represents the peak value at the frequency $\omega_{2}$ (see Figure 2). The proposed model depends on 5 parameters, ${ }_{103}$ namely $G_{0}$ and the four exponents $e_{i}(i=1, \cdots, 4)$, whose closed-form expressions have been derived by manipulating 104 Eq. (3):

$$
\begin{gathered}
e_{1}=2 k_{2}-1-L\left(\omega_{1}\right) ; \\
e_{2}=2 k_{1}-1-L\left(\omega_{2}\right) ; \\
e_{3}=-1-\gamma-\beta_{2} L\left(\omega_{2}\right) ; \\
{ }_{106} \quad e_{4}=-1-\gamma-\beta_{3}\left(L\left(\omega_{3}\right)+2 \frac{\alpha-1}{\alpha}\right) ; \\
{ }_{108} \\
G_{0}=\frac{\gamma}{\beta_{2} \omega_{2}}\left(\frac{\alpha S_{0}}{\eta_{U}\left(\omega_{2}, \zeta\right)}\right)^{2} .
\end{gathered}
$$

109 In Eqs. (6) to (9), the function $L(\omega)$ is defined as:

$$
L(\omega)=2 \omega \frac{\mathrm{d}}{\mathrm{d} \omega} \ln \left(\eta_{U}(\omega, \zeta)\right)
$$

110 and the following quantities have been introduced:

$$
\beta_{2}=\left(\frac{\omega_{1}}{\omega_{2}}\right)^{e_{2}+1} \frac{\gamma+e_{1}+1}{e_{1}+1}+\left(1-\left(\frac{\omega_{1}}{\omega_{2}}\right)^{e_{2}+1}\right) \frac{\gamma+e_{2}+1}{e_{2}+1}
$$




$$
\beta_{3}=\left(\frac{\omega_{2}}{\omega_{3}}\right)^{e_{3}+1} \beta_{2}+\left(1-\left(\frac{\omega_{2}}{\omega_{3}}\right)^{e_{3}+1}\right) \frac{\gamma+e_{3}+1}{e_{3}+1} .
$$

112 For the sake of completeness, the mathematical process leading to Eqs. (6) to (13) is detailed in Appendix A. It is 113 worth stressing here that Eqs. (6) to (13) map the $5 \mathrm{RS}$ parameters $\alpha, S_{0}, T_{1}=2 \pi / \omega_{1}, T_{2}=2 \pi / \omega_{2}$ and $T_{3}=2 \pi / \omega_{3}$ 114 onto the 5 PSD parameters $G_{0}, e_{1}, e_{2}, e_{3}$ and $e_{4}$ through explicit analytical relationships. Accordingly, the definition 115 of the seismic action can be equivalently done in terms of RS or PSD function.

${ }_{116}$ The PSD function of Eq. (5) can be easily adapted to 3- or 2-branch RS. In the first case, the constant displacements 117 branch should simply be removed, obtaining the corresponding RS by letting $T_{1} \rightarrow \infty$ in Eq. (2). Hence, the RS118 consistent PSD function can be derived by letting $\omega_{1} \rightarrow 0$ in Eq. (5):

$$
S_{\mathrm{pa}}(T)= \begin{cases}S_{0}\left[1+(\alpha-1) \frac{T}{T_{3}}\right], & 0 \leq T<T_{3} ; \\ \alpha S_{0}, & T_{3} \leq T \leq T_{2} ; \\ \alpha S_{0}\left(\frac{T_{2}}{T}\right)^{k_{1}}, & T>T_{2} ;\end{cases}
$$

119

$$
G_{\ddot{U}_{\mathrm{g}}}(\omega)= \begin{cases}G_{0}\left(\frac{\omega}{\omega_{2}}\right)^{e_{2}}, & 0 \leq \omega<\omega_{2} ; \\ G_{0}\left(\frac{\omega}{\omega_{2}}\right)^{e_{3}}, & \omega_{2} \leq \omega \leq \omega_{3} ; \\ G_{0}\left(\frac{\omega_{3}}{\omega_{2}}\right)^{e_{3}}\left(\frac{\omega}{\omega_{3}}\right)^{e_{4}}, & \omega>\omega_{3} .\end{cases}
$$

${ }_{120}$ For 2-branch RS, the initial linear branch is removed as well, letting $T_{3} \rightarrow 0$ and $\omega_{3} \rightarrow \infty$ in Eq. (14), and returning ${ }_{121}$ the expressions:

122

$$
\begin{gathered}
S_{\mathrm{pa}}(T)=\left\{\begin{array}{lc}
\alpha S_{0}, & T \leq T_{2} ; \\
\alpha S_{0}\left(\frac{T_{2}}{T}\right)^{k_{1}}, & T>T_{2} ;
\end{array}\right. \\
G_{\ddot{U}_{\mathrm{g}}}(\omega)= \begin{cases}G_{0}\left(\frac{\omega}{\omega_{2}}\right)^{e_{2}}, & 0 \leq \omega<\omega_{2} ; \\
G_{0}\left(\frac{\omega}{\omega_{2}}\right)^{e_{3}}, & \omega \geq \omega_{2} .\end{cases}
\end{gathered}
$$

${ }_{123}$ Also in these cases, the exponents $e_{i}$ and $G_{0}$ can be derived using Eqs. (7) to (10).

\section{3. Analytical spectral moments of the response process}

${ }_{125}$ Spectral moments play an important role in random vibration problems, as they are related to various statistics of 126 stochastic processes (e.g. variance of its time derivatives, central frequency, bandwidth parameter, etcetera) and allow ${ }_{127}$ deriving approximate solutions for the first-passage problem [33-38, 48, 49]. Mathematically defined as the geometric 128 moments of the one-sided PSD function $G_{X}(\omega)$ with respect to the axis $\omega=0$, the generic $m$ th order spectral moment 129 of a stochastic process $X(t)$ is given by:

$$
\lambda_{m, X}=\int_{0}^{+\infty} \omega^{m} G_{X}(\omega) \mathrm{d} \omega
$$

${ }_{130}$ In general, the integral in Eq. (16) can only be computed numerically. In this paper, however, closed-form expres${ }_{131}$ sions of the spectral moments for structures excited by RS-consistent earthquake acceleration processes are proposed, 
${ }_{132}$ in approximate form, for order $m$ up to three for SDOF oscillators and up to two for MDOF structures. To the best ${ }_{133}$ of the authors' knowledge, spectral moments in analytical form have never been proposed before for RS-consistent ${ }_{134}$ seismic accelerations.

\section{3.1. Spectral moments for SDOF systems}

${ }_{136}$ Let us consider a linear SDOF oscillator driven by a base acceleration time history $\ddot{u}_{\mathrm{g}}(t)$, sample of a process $\ddot{U}_{\mathrm{g}}(t)$, ${ }_{137}$ fully characterized by the PSD function $G_{\ddot{U}_{\mathrm{g}}}(\omega)$, consistent with an assigned RS. Based on Eq. (16), the $m$ th-order 138 spectral moment of the response process can be written as:

$$
\lambda_{m, U}=\int_{0}^{+\infty} \omega^{m}|H(\omega)|^{2} G_{\ddot{U}_{\mathrm{g}}}(\omega) \mathrm{d} \omega,
$$

${ }_{139}$ where $H(\omega)$ is the complex-valued transfer function of the SDOF oscillator:

$$
H(\omega)=\frac{1}{\omega_{0}^{2}-\omega^{2}+2 \imath \zeta \omega_{0} \omega},
$$

140 in which $\omega_{0}$ and $\zeta$ are the natural circular frequency and the viscous damping ratio of the system, while $\imath=\sqrt{-1}$ is 141 the imaginary unit.

${ }_{142}$ A first approximation of the spectral moments can be obtained if the function $|H(\omega)|^{2}$ exhibits a very sharp peak ${ }_{143}$ in $\omega_{0}$ (e.g., damping ratio $\zeta \ll 1$ ) and the PSD function of the excitation is approximately flat in the neighbourhood ${ }_{144}$ of $\omega_{0}$. In this case, the spectral moments of order $m \leq 3$ can be determined in closed-form considering the input as a 145 white noise process with constant PSD function $G_{\ddot{U}_{\mathrm{g}}}\left(\omega_{0}\right)$ (see, for instance, Ref. [50]):

146

$$
\lambda_{0, U}^{(1)}=\frac{\pi}{4 \zeta \omega_{0}^{3}} G_{\ddot{U}_{\mathrm{g}}}\left(\omega_{0}\right)
$$

$$
\begin{gathered}
{ }^{146} \lambda_{1, U}^{(1)}=\frac{\pi-2 \zeta}{4 \zeta \omega_{0}^{2}} G_{\ddot{U}_{\mathrm{g}}}\left(\omega_{0}\right) ; \\
{ }_{147} \lambda_{2, U}^{(1)}=\omega_{0}^{2} \lambda_{0, U}^{(1)} ; \\
\lambda_{3, U}^{(1)}=\omega_{0}^{2} \lambda_{1, U}^{(1)},
\end{gathered}
$$

149 where the superscripted (1) serves as a reminder of being the "first" approximation for the spectral moments.

150 An improvement to this simplified approach [35] entails accounting for contributions in the frequency range ${ }_{151}\left[0, \omega_{0}\right]$, where the function $|H(\omega)|^{2}$ can be reasonably approximated as $1 / \omega_{0}^{4}$. In this case, the spectral moments of ${ }_{152}$ order $m \leq 3$ can be calculated as:

$$
\lambda_{m, U}^{(2)}=\lambda_{m, U}^{(1)}+\frac{1}{\omega_{0}^{4}} \int_{0}^{\omega_{0}} G_{\ddot{U}_{\mathrm{g}}}(\omega) \mathrm{d} \omega-\frac{G_{\ddot{U}_{\mathrm{g}}}\left(\omega_{0}\right)}{(m+1) \omega_{0}^{3-m}},
$$

${ }_{153}$ where $\lambda_{m, U}^{(1)}$ are the expressions in Eq. (19), the negative terms in the right hand side takes away the contribution around ${ }_{154} \omega=\omega_{0}$, already included in $\lambda_{m, U}^{(1)}$, and the superscripted (2) means "second" approximation. Taking advantage of the 155 analytical PSD model proposed in Section 2.2, these expressions can be rewritten as:

$$
\lambda_{m, U, k}^{(2)}=\frac{G_{\ddot{U}_{\mathrm{g}}}\left(\omega_{0}\right)}{4 \zeta \omega_{0}^{3-m}} \varphi_{m, k}+\frac{1}{\omega_{0}^{4}} \sum_{j=1}^{k-1} G_{\ddot{U}_{\mathrm{g}}}\left(\omega_{j}\right) \omega_{j}^{m+1} \gamma_{m, j},
$$

${ }_{156}$ where $\lambda_{m, U, k}^{(2)}$ is the expression of the $m$-th spectral moment of the response process $U(t)$ when $\omega_{0}$ falls within the $k$ th 157 branch of Eq. (5), while the dimensionless quantities $\varphi_{m, k}$ and $\gamma_{m, j}$ are defined as follows:

$$
\varphi_{m, k}=\pi-\frac{4 \zeta}{(1+m)\left(1+m+e_{k}\right)} \begin{cases}e_{k}, & m=0,2 ; \\ 2\left(1+e_{k}\right), & m=1 ; \\ 8+3 e_{k}, & m=3 ;\end{cases}
$$




$$
\gamma_{m, j}=\frac{e_{j+1}-e_{j}}{\left(1+m+e_{j+1}\right)\left(1+m+e_{j}\right)} .
$$

159 Eq. (21) is valid for 4-branch RS, and can be easily extended to the case of 2 and 3 branches cases. In fact, 160 following the notation adopted in Eqs. (14) and (15), for the 3-branch $\mathrm{RS}(2<k<4)$, the lower bound in the 161 summation must be changed to $j=2$, while for the 2-branch case $(2<k<3)$ lower and upper bounds should be 162 modified to $j=2$ and $k=2$, respectively.

${ }_{163}$ Eq. (21) constitutes an accurate estimate of the spectral moments as a closed-form function of the RS parameters. ${ }_{164} \mathrm{It}$ is worth noting that, by setting all the exponents $e_{i}$ in Eq. (5) to zero, the PSD function assumes constant value $G_{0}$ 165 and Eq. (21) returns the well-known spectral moments for white noise input.

\section{3.2. Spectral moments for MDOF systems}

${ }_{167}$ The equations of motion of a $n$-degree-of-freedom system under seismic action can be expressed as:

$$
\mathbf{M} \cdot \ddot{\mathbf{u}}(t)+\mathbf{C} \cdot \dot{\mathbf{u}}(t)+\mathbf{K} \cdot \mathbf{u}(t)=-\mathbf{M} \cdot \boldsymbol{\tau} \ddot{u}_{\mathrm{g}}(t),
$$

${ }_{168}$ where $\mathbf{M}, \mathbf{C}$ and $\mathbf{K}$ are the $(n \times n)$ time-invariant mass, damping and stiffness matrices, respectively; $\mathbf{u}(t)$ is the $(n \times 1)$ 169 vector listing the degrees of freedom of the structure; and $\tau$ is the $(n \times 1)$ location vector.

Closed-form direct and cross spectral moments for classically-damped MDOF system can be determined by first 171 introducing the modal matrix $\boldsymbol{\Phi}$ of the system, solution of the classical eigen-problem $\mathbf{K} \cdot \boldsymbol{\Phi}=\mathbf{M} \cdot \boldsymbol{\Phi} \cdot \boldsymbol{\Omega}^{2}$, with 172 the ortho-normal condition $\boldsymbol{\Phi}^{\mathrm{T}} \cdot \mathbf{M} \cdot \boldsymbol{\Phi}=\mathbf{I}_{n}, \mathbf{I}_{n}$ being the identity matrix of size $n$, and then applying the modal 173 transformation $\mathbf{u}(t)=\boldsymbol{\Phi} \cdot \mathbf{y}(t)$, leading to the following equation:

$$
\ddot{\mathbf{y}}(t)+\boldsymbol{\Gamma} \cdot \dot{\mathbf{y}}(t)+\mathbf{\Omega}^{2} \cdot \mathbf{y}(t)=\mathbf{p} \ddot{u}_{\mathrm{g}}(t),
$$

174 where $\mathbf{y}(t)$ is the vector collecting the $\ell \leq n$ modal coordinates retained in the analysis; $\boldsymbol{\Gamma}=\operatorname{diag}\left\{\begin{array}{llll}2 \zeta_{1} \omega_{1} & \cdots & 2 \zeta_{\ell} \omega_{\ell}\end{array}\right\}$ 175 and $\boldsymbol{\Omega}=\operatorname{diag}\left\{\begin{array}{lll}\omega_{1} & \cdots & \omega_{\ell}\end{array}\right\}$ are the modal damping matrix and spectral matrix, respectively, both of dimensions ${ }_{176}(\ell \times \ell)$; and $\mathbf{p}=-\boldsymbol{\Phi}^{\mathrm{T}} \cdot \mathbf{M} \cdot \tau=\left\{\begin{array}{lll}p_{1} & \cdots & p_{\ell}\end{array}\right\}^{\mathrm{T}}$ is the vector of modal participation factors. Under the assump177 tions of Gaussian excitation, cross-spectral moments of $m$ th order related to the $j$ th and $k$ th modal oscillators (with $178 j=1, \cdots, \ell$ and $k=1, \cdots, \ell)$ can be defined as:

$$
\lambda_{m, j, k}=\int_{0}^{+\infty} \omega^{m} p_{j} p_{k} H_{j}^{*}(\omega) H_{k}(\omega) G_{\ddot{U}_{\mathrm{g}}}(\omega) \mathrm{d} \omega
$$

179 where the asterisk means complex conjugate and $H_{j}(\omega)$ is the transfer function of the $j$ th modal oscillator, so defined:

$$
H_{j}(\omega)=\frac{1}{\omega_{j}^{2}-\omega^{2}+2 \iota \zeta_{j} \omega_{j} \omega} .
$$

${ }_{180}$ For a selected RS, the direct spectral moments $(j=k)$ can be estimated using Eq. (21). Then, as demonstrated by ${ }_{181}$ Di Paola and Muscolino [38], the cross-spectral moments of any order, if they exist for a given PSD function of the 182 excitation, can be obtained recursively as linear combinations of the direct spectral moments. In particular, real and 183 imaginary parts of even-order cross-spectral moments $(m=0,2)$ can be determined as:

$$
\operatorname{Re}\left[\lambda_{m, j, k}\right]=\frac{(-1)^{m / 2}}{2}\left(\lambda_{0, j, j} \gamma_{m, k, j} \omega_{j}^{2}+\lambda_{2, j, j} \delta_{m, k, j}+\lambda_{0, k, k} \gamma_{m, j, k} \omega_{k}^{2}+\lambda_{2, k, k} \delta_{m, j, k}\right)
$$

184

$$
\operatorname{Im}\left[\lambda_{m, j, k}\right]=\frac{(-1)^{m / 2}}{2}\left(\lambda_{1, j, j} \varepsilon_{m, k, j}-\lambda_{1, k, k} \varepsilon_{m, j, k}+\lambda_{3, j, j} \alpha_{m, j, k}-\lambda_{3, k, k} \alpha_{m, k, j}\right),
$$

185 while for the first-order cross-spectral moments:

$$
\operatorname{Re}\left[\lambda_{1, j, k}\right]=-\frac{1}{2}\left(\lambda_{1, j, j} \varepsilon_{1, k, j}+\lambda_{1, k, k} \varepsilon_{1, j, k}+\lambda_{3, j, j} \alpha_{1, k, j}+\lambda_{3, k, k} \alpha_{1, j, k}\right) ;
$$




$$
\operatorname{Im}\left[\lambda_{1, j, k}\right]=\frac{1}{2}\left(\lambda_{0, j, j} \gamma_{1, k, j} \omega_{j}^{2}+\lambda_{2, j, j} \delta_{1, k, j}-\lambda_{0, k, k} \gamma_{1, j, k} \omega_{k}^{2}-\lambda_{2, k, k} \delta_{1, j, k}\right) .
$$

192 being:

193

194

195

In Eqs. (28) and (29), the following positions have been made:

$$
\begin{gathered}
\alpha_{m, j, k}=\alpha_{m-1, j, k}+\bar{\omega}_{k} \beta_{m-1, j, k} ; \\
\beta_{m, j, k}=-\zeta_{k} \omega_{k} \beta_{m-1, j, k}-\bar{\omega}_{k} \alpha_{m-1, j, k} ; \\
\gamma_{m, j, k}=\zeta_{k} \omega_{k} \alpha_{m, j, k}+\bar{\omega}_{k} \beta_{m, j, k} ; \\
\delta_{m, j, k}=\zeta_{k} \omega_{k} \alpha_{m, j, k}-\bar{\omega}_{k} \beta_{m, j, k} ; \\
\varepsilon_{m, j, k}=2 \zeta_{k} \omega_{k} \bar{\omega}_{k} \beta_{m, j, k}+\alpha_{m, j, k}\left(\omega_{k}^{2}-2 \bar{\omega}_{k}^{2}\right) ;
\end{gathered}
$$

196 Once the matrix of the cross-spectral moments of order $m$ of the modal oscillators, $\boldsymbol{\Lambda}_{m, \mathbf{Y}}$, is obtained, the corre${ }_{197}$ sponding quantities in the geometric space can be computed as:

$$
\boldsymbol{\Lambda}_{m, \mathbf{X}}=\boldsymbol{\Phi} \cdot \boldsymbol{\Lambda}_{m, \mathbf{Y}} \cdot \boldsymbol{\Phi}^{\mathrm{T}} .
$$

${ }_{198}$ It is worth noting here that, when the seismic peak response of MDOF structures is of interest, as for instance in the 199 case-study structure discussed in the Section 5, cross-spectral moments up to second order are required, and thus the 200 computation of direct spectral moments up to third order is needed. Importantly, for the proposed spectrum-consistent ${ }_{201}$ PSD function of the ground shaking, they all exist and can be determined using the expression reported above.

\section{4. Numerical validation for SDOF oscillators}

${ }_{203}$ The proposed approach provides closed-form expressions for the statistics of the seismic response of linear structures, 204 inevitably within the approximations associated with the analytical model for the PSD function of the input and the 205 hypotheses underpinning Eqs. (19) and (20). Therefore, the accuracy of the method is first tested in this section for ${ }_{206}$ SDOF oscillators, through: $i$ ) comparison with the results of numerical integration for the first three spectral moments ${ }_{207} \lambda_{m}, U$ for different values of the natural period of vibration and viscous damping ratio; ii) consistency analysis for 208 three different international building codes, adopting RS with different numbers of branches; iii) seismic reliability 209 analysis for SDOF oscillators with different natural periods of vibration.

\section{4.1. Spectral moments for SDOF oscillators}

${ }_{211}$ In this section, a number of linear SDOF oscillators are analyzed, varying their natural periods of vibration and viscous ${ }_{212}$ damping ratios. A pseudo-acceleration RS has been selected according to the Italian Code [51] (which uses the ${ }_{213}$ same 3-branch shape as Eurocode 8, and whose parameters are defined through seismic maps and vary continuously 214 with latitude and longitude), considering a return period of 475 years (i.e., the so-called "no-collapse requirement", ${ }_{215}$ associated with $10 \%$ probability of exceedance in 50 years) and a site in Messina (Sicily), with "type A" soil (hard ${ }_{216}$ rock). Table 2 reports the parameters of both the RS and the RS-consistent PSD obtained through the application of ${ }_{217}$ Eqs. (5) to (13).

${ }_{218}$ In Figure 3, closed-form spectral moments of order $m=0,1$ and 2 are compared with their numerical counterpart 219 (computed by numerical integration of the unilateral PSD of the dynamic response, $G_{U}(\omega)=|H(\omega)|^{2} G_{\ddot{U}_{\mathrm{g}}}(\omega)$ ) for 220 a range of natural periods of vibration and viscous damping ratios. Results are in excellent agreement, with only 
Table 2: Parameters for the definition of the four-branch RS and PSD.

\begin{tabular}{cccc}
\hline \multicolumn{2}{c}{ RS } & \multicolumn{2}{c}{ PSD } \\
\hline parameter & value & parameter & value \\
\hline$S_{0}$ & $2.432 \mathrm{~m} / \mathrm{s}^{2}$ & $G_{0}$ & $1.4513 \cdot 10^{-2}\left(\mathrm{~m} / \mathrm{s}^{2}\right)^{2} /(\mathrm{rad} / \mathrm{s})$ \\
$\alpha$ & 2.411 & $e_{1}$ & 2.5094 \\
$T_{3}$ & $0.120 \mathrm{~s}$ & $e_{2}$ & 0.7594 \\
$T_{2}$ & $0.359 \mathrm{~s}$ & $e_{3}$ & -1.3177 \\
$T_{1}$ & $2.592 \mathrm{~s}$ & $e_{4}$ & -2.6209 \\
\hline
\end{tabular}

${ }_{221}$ relatively small discrepancies observed for high-order moments at large natural periods and damping ratios. For 222 instance, for damping ratios less than $10 \%$ and periods of vibration less than $2 \mathrm{~s}$, deviations are less than $10 \%$. For 223 larger damping ratios and periods of vibration (i.e. relatively high-damped flexible structures) the proposed approach 224 tends to underestimate the spectral moments of the dynamic response, mainly due to an insufficiently long duration 225 assumed for the stationary excitation. In order to achieve higher accuracy, this well-known problem can be addressed 226 by adopting Vanmarcke's frequency-dependent corrective damping factor [35]. Alternatively, depending on the natural ${ }_{227}$ period of vibration and viscous damping ratio of the structure being analyzed, a sufficiently long duration of the ${ }_{228}$ stationary excitation could be assumed. The latter can be selected on the basis of the numerical results reported in 229 Ref. [40].

\section{4.2. Reproduction of the target response spectra}

${ }_{231}$ Eurocode 8 [7] provides the requirements for alternative representations of the seismic action. In particular, according ${ }_{232}$ to clause $\S 3.2 .3 .1 .2$, a suite of artificial accelerograms can be utilized if, in the range of periods between $0.2 T_{0}$ ${ }_{233}$ and $2 T_{0}$ (where $T_{0}$ is the fundamental period of the structure in the direction to which the accelerogram is applied), ${ }_{234}$ no values of the mean 5\%-damping $\operatorname{RS~} \hat{S}_{\mathrm{pa}}(T)$, calculated from all the response time histories, is less than $90 \%$ of 235 the corresponding value of the targeted 5\%-damping design $\operatorname{RS} S_{\mathrm{pa}}(T)$; that is, $\hat{S}_{\mathrm{pa}}(T) \geq 0.90 S_{\mathrm{pa}}(T)$. In a stochastic 236 framework, this corresponds to a consistency criterion between PSD and RS where the 50th percentile (i.e. the median) ${ }_{237}$ of the peak dynamic response of any SDOF oscillator with natural period $T_{0}=2 \pi / \omega_{0}$, excited by a stationary random 238 process with given PSD function, exceeds $90 \%$ of the targeted 5\%-damping design RS.

${ }_{239}$ For validation purposes, three PSD functions consistent with RS defined by three different building codes have 240 been considered. For each period of vibration $T, \hat{S}_{\mathrm{pa}}(T)$ can be estimated as the median of the maximum response in 241 terms of pseudo-accelerations:

$$
\hat{S}_{\mathrm{pa}}(T)=\left(\frac{2 \pi}{T}\right)^{2} \sqrt{\lambda_{0, U}(T, \zeta)} \eta_{U}\left(\frac{2 \pi}{T}, \zeta\right),
$$

242 where $\lambda_{0, X}(T, \zeta)$ is the zero-order spectral moment of the response process $U(t)$ and $\eta_{U}(\omega, \zeta)$ is the corresponding 243 Vanmarcke's peak factor:

$$
\eta_{U}(\omega, \zeta)=\sqrt{2 \ln \left\{2 N_{U}(\omega, \zeta)\left[1-\exp \left(-q_{U}^{1.2}(\omega, \zeta) \sqrt{\pi \ln \left(2 N_{U}(\omega, \zeta)\right)}\right)\right]\right\}}
$$

244 being:

245

$$
N_{U}(\omega, \zeta)=\frac{T_{\mathrm{S}} v_{U}(\omega, \zeta)}{-\ln (p)}
$$

$$
v_{U}(\omega, \zeta)=\frac{1}{2 \pi} \sqrt{\frac{\lambda_{2, U}(2 \pi / \omega, \zeta)}{\lambda_{0, U}(2 \pi / \omega, \zeta)}}
$$

246

$$
q_{U}(\omega, \zeta)=\sqrt{1-\frac{\lambda_{1, U}^{2}(2 \pi / \omega, \zeta)}{\lambda_{0, U}(2 \pi / \omega, \zeta) \lambda_{2, U}(2 \pi / \omega, \zeta)}},
$$

${ }_{247}$ where $v_{U}(\omega, \zeta)$ and $q_{U}(\omega, \zeta)$ are the mean up-crossing rate and the bandwidth factor of the random process $U(t)$. 

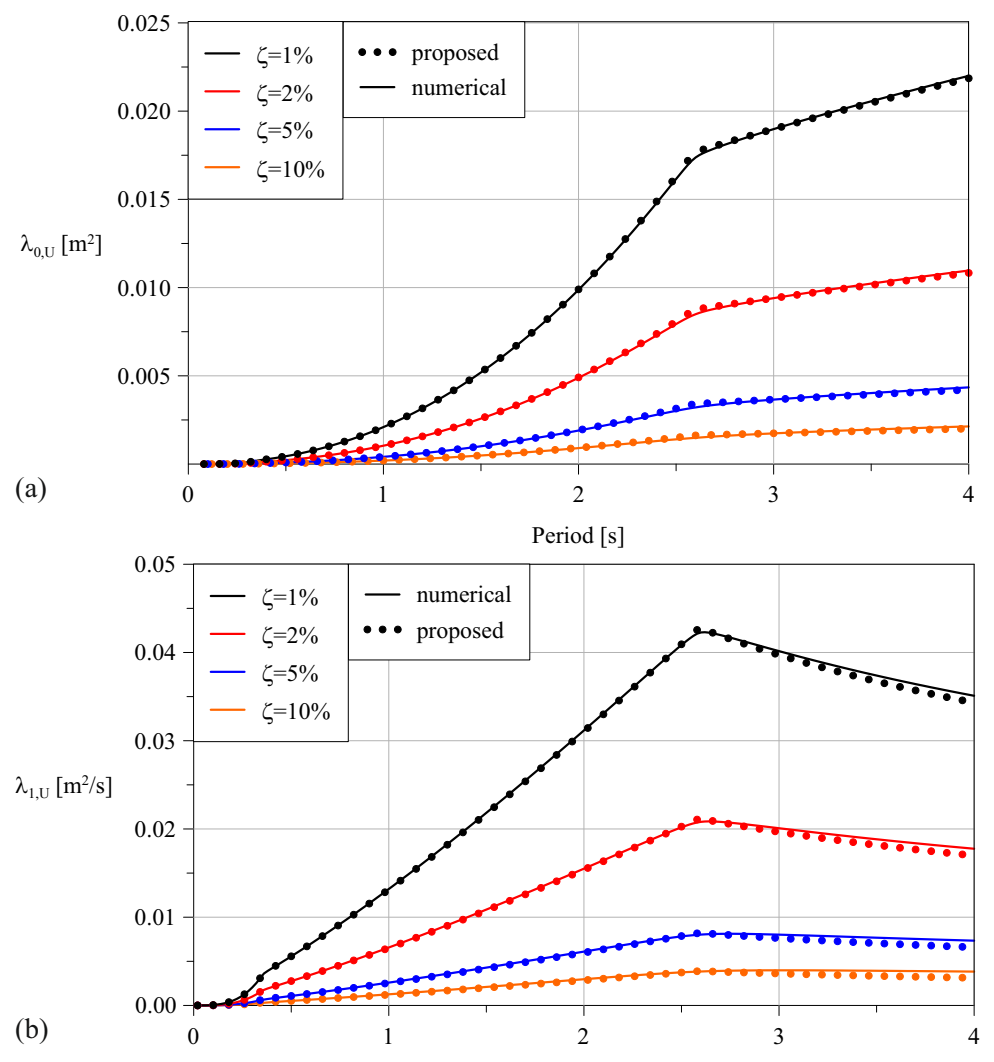

(b)

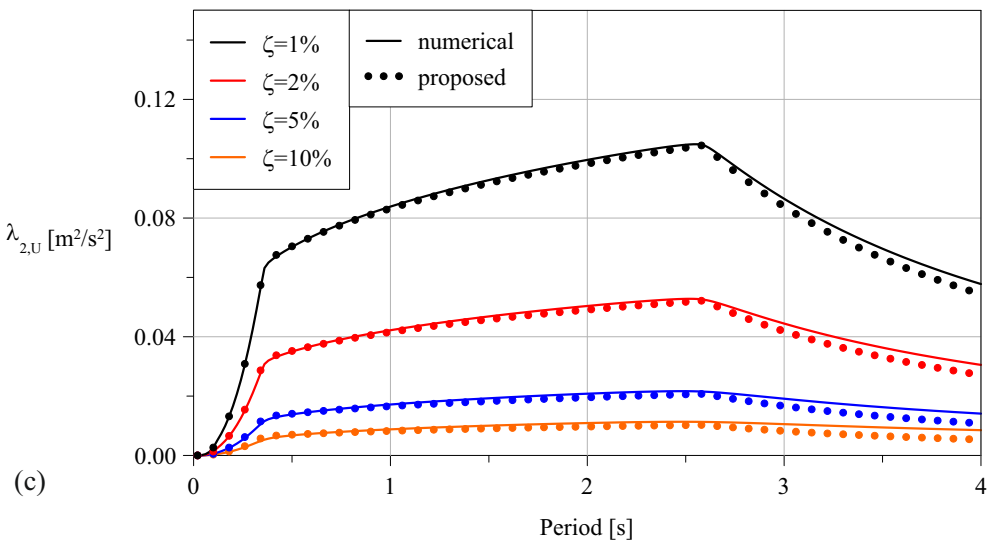

Figure 3: Comparison between numerical evaluation (solid lines) and proposed approximate analytic expressions (dots) of spectral moments against natural period; a) zero order moments; b) first order moments; c) second order moments. 


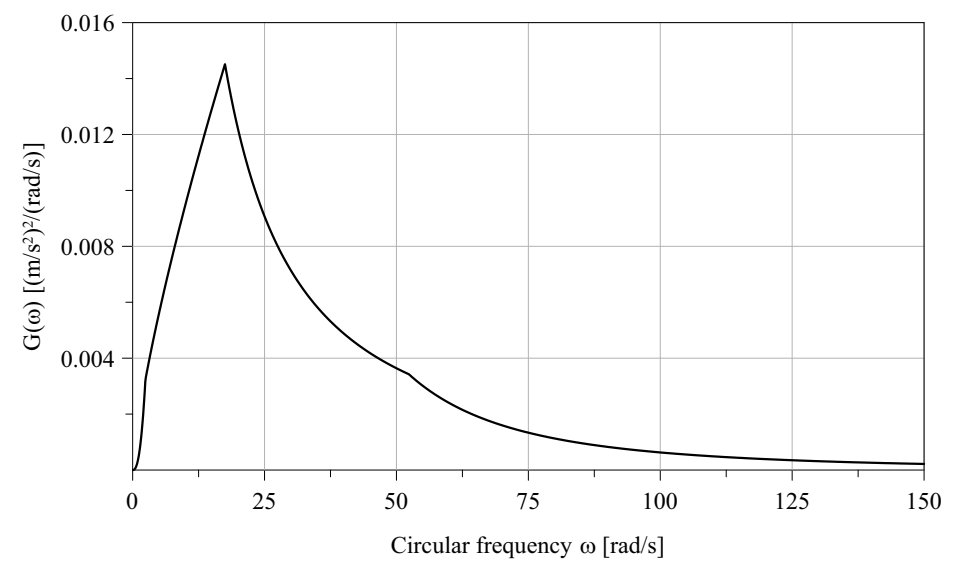

(a)

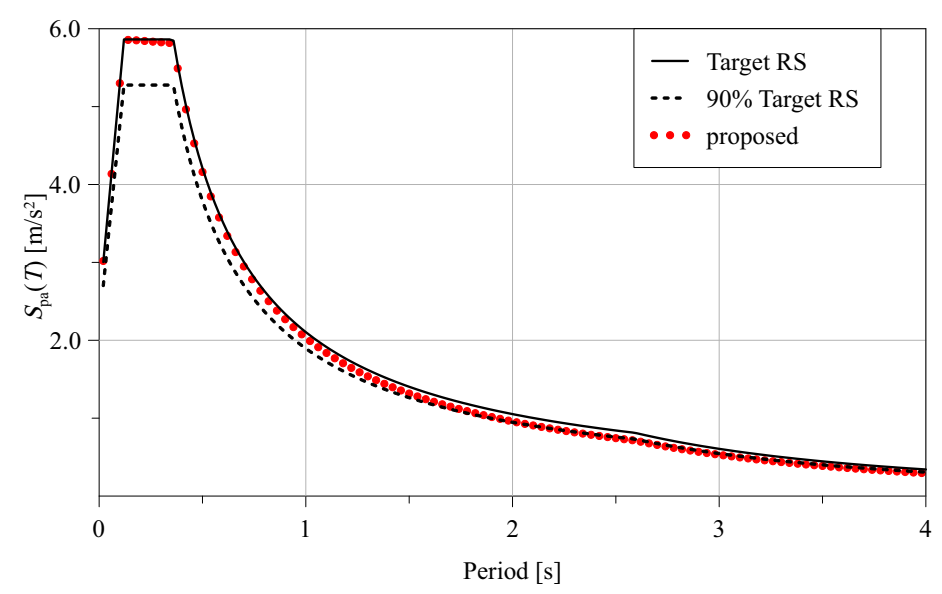

(b)

Figure 4: (a) PSD function consistent with the four-branch RS (parameters listed in Table 2; (b) Pseudo-acceleration RS: target (black solid line), $10 \%$ tolerance (black dashed line) and generated RS obtained with proposed approach (red dots).

248 It should be noted at this point that the quantities of Eqs. (35) (and, ultimately, the peak factor of Eq. (34)) depend 249 on: $i$ ) the spectral moments $\lambda_{m, U}$ up to the order $m=2$, which can be calculated with of Eq. (21); ii) the fractile $p$ 250 of the maxima distribution, set $p=0.50$ (i.e. median of the distribution); iii) the duration $T_{\mathrm{S}}$ of the stationary part ${ }_{251}$ of the input, which in the following has been arbitrarily assumed as $T_{\mathrm{S}}=20 \mathrm{~s}$. Obviously, the larger $T_{S}$, the lower 252 the ordinates of the PSD function of the seismic input, but the same peak response is obtained for linear structural 253 systems.

${ }_{254}$ The 4-branch pseudo-acceleration RS, with parameters reported in Table 2, has been considered first. Figure 4(a) 255 shows the RS-consistent PSD obtained with the application of Eqs. (5) to (13), while Figure 4(b) compares the code 256 and reconstructed RS in terms of pseudo-accelerations, showing that the latter lies within the allowed $10 \%$ tolerance. 257 A 3-branch pseudo-acceleration RS has then been considered, following the International Building Code [46] and 258 assuming that the SDOF oscillator was representative of structure located in Memphis (Missouri, USA), on a type A 259 soil (hard rock). The RS-consistent PSD function is shown in Figure 5(a), whose parameters are reported in Table 3. ${ }_{260}$ Also in this case, the discrepancy between the reconstructed and target RS does not exceed $10 \%$ for all periods of 261 vibration, as illustrated in Figure 5(b).

${ }_{262}$ Finally, the two-branch RS defined by the Peruvian building code [47], with reference to an ordinary building 263 located in the country's second seismic zone, on soil "type S1" (hard rock). The parameters of RS and PSD are ${ }_{264}$ reported in Table 4, and the RS-consistent PSD function is depicted in Figure 6(a), and the results in terms of pseudo265 acceleration RS are shown in Figure 6(b).

${ }_{266}$ Although not reported herein for sake of shortness, similar results have been obtained for all the international ${ }_{267}$ codes listed in Table 1, confirming thus the validity of the proposed approach.

\section{4.3. Reliability analysis}

${ }_{269}$ The statistical distribution of the peak structural response can play a fundamental role in the seismic analysis and 270 design of multi-story frames. Herein, the distribution of the maxima of a response process is determined by coupling 271 the proposed formulation with the approach presented by Vanmarcke in Ref. [34], since the two techniques are based 272 on common underpinning assumptions. This allows exploiting the significant computational advantages of the closed273 form spectral moments of the response process. Results for SDOF oscillators are presented in this section, and 274 compared with the outcomes of Monte Carlo simulations. The extension to MDOF structures is specifically addressed 275 in Section 5.

276 Based on Ref. [34], for a stationary random process $U(t)$ (e.g. the displacements of a structure, relative to the ${ }_{277}$ ground), the probability associated to the non-occurrence of crossings of a generic barrier $b$ into the time window $T_{\mathrm{S}}$ 
Table 3: Parameters for the definition of the three-branch RS and PSD.

\begin{tabular}{cccc}
\hline \multicolumn{2}{c}{ RS } & \multicolumn{2}{c}{ PSD } \\
\hline parameter & value & parameter & value \\
\hline$S_{0}$ & $1.902 \mathrm{~m} / \mathrm{s}^{2}$ & $G_{0}$ & $9.2629 \cdot 10^{-3}\left(\mathrm{~m} / \mathrm{s}^{2}\right)^{2} /(\mathrm{rad} / \mathrm{s})$ \\
$\alpha$ & 2.500 & $e_{2}$ & 0.7609 \\
$T_{3}$ & $0.070 \mathrm{~s}$ & $e_{3}$ & -1.3164 \\
$T_{2}$ & $0.351 \mathrm{~s}$ & $e_{4}$ & -2.7216 \\
\hline
\end{tabular}

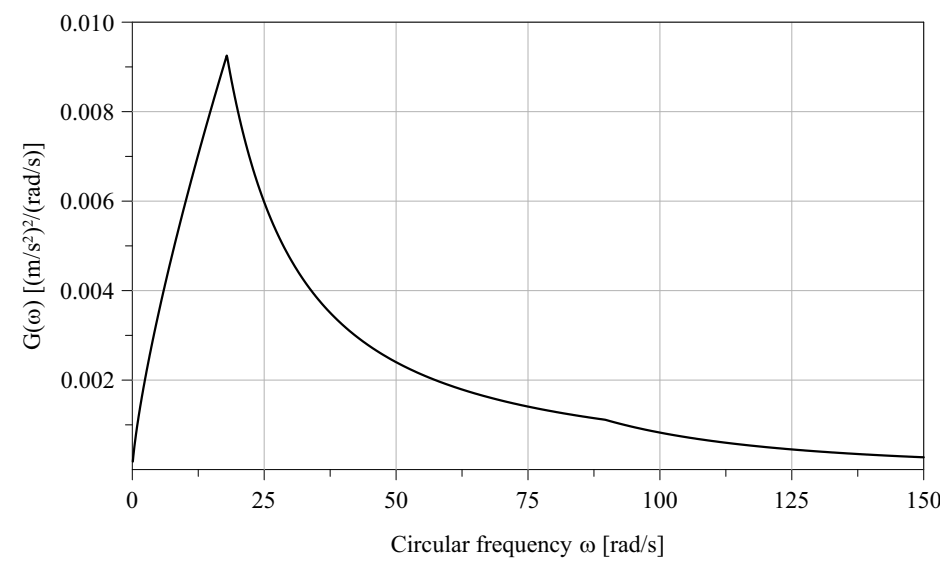

(a)

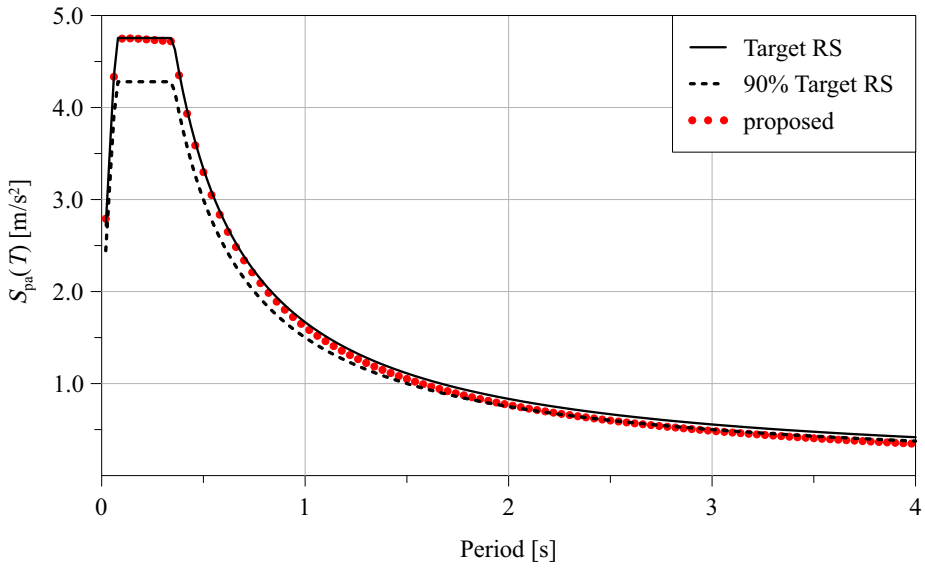

(b)

Figure 5: (a) PSD function consistent with the three-branch RS (parameters listed in Table 3; (b) Pseudo-acceleration RS: target (black solid line), $10 \%$ tolerance (black dashed line) and generated RS obtained with proposed approach (red dots).

278 can be evaluated as:

$$
P_{\mathrm{S}}\left(b, T_{\mathrm{S}}\right)=1-\exp \left(-\frac{b^{2}}{\lambda_{0, U}}\right) \exp \left(-\tilde{\alpha}_{U}(b) T_{\mathrm{S}}\right),
$$

${ }_{279}$ where $\tilde{\alpha}_{U}$ is the so-called "risk function" for the random process $U(t)$, defined as:

$$
\tilde{\alpha}_{U}(b)=2 v_{U} \frac{1-\exp \left(-\sqrt{\frac{\pi}{2 \lambda_{0, U}}} b q_{U}^{1.2}\right)}{\exp \left(\frac{b^{2}}{2 \lambda_{0, U}}-1\right)}
$$

280 in which the mean zero-crossing rate $v_{U}=v_{U}(\omega, \zeta)$ and the bandwidth factor $q_{U}=q_{U}(\omega, \zeta)$ have been defined in ${ }_{281}$ Eqs. (35b) and (35c), respectively. Note that, to simplify the notation, the explicit dependency on the properties of the ${ }_{282}$ SDOF oscillator, namely $T=2 \pi / \omega$ and $\zeta$, has been omitted in Eqs. (36) and (37).

${ }_{283}$ Importantly, since only the spectral moments of order up to two are involved, full statistical distribution of the peak 284 values of the process can be determined in closed-form by using the analytical expressions proposed in Section 3.

285 For the case of SDOF oscillators and RS-consistent PSD functions depicted in Figure 3 (and parameters listed in ${ }_{286}$ Table 2), the proposed approach has been applied to determine the cumulative distribution function (CDF) of the peak ${ }_{287}$ values of the displacement process $U(t)$, varying the natural period of vibration of the system. Results, computed ${ }_{288}$ considering an observation time $T_{\mathrm{S}}=20 \mathrm{~s}$, are compared in Figure 7 with the outcomes of Monte Carlo simulations 289 (MCS). The latter have been performed with 20,000 samples of ground acceleration, generated as realizations of a 290 stationary, zero-mean Gaussian process, fully defined by the proposed PSD function. In order to analyze station291 ary response processes within the time observation window $T_{\mathrm{S}}$, the time modulation function proposed by Jennings 
Table 4: Parameters for the definition of the two-branch RS and PSD.

\begin{tabular}{cccc}
\hline \multicolumn{2}{c}{ RS } & \multicolumn{2}{c}{ PSD } \\
\hline parameter & value & parameter & value \\
\hline$S_{0}$ & $2.941 \mathrm{~m} / \mathrm{s}^{2}$ & $G_{0}$ & $2.6098 \cdot 10^{-2}\left(\mathrm{~m} / \mathrm{s}^{2}\right)^{2} /(\mathrm{rad} / \mathrm{s})$ \\
$\alpha$ & 2.500 & $e_{2}$ & 0.7526 \\
$T_{2}$ & $0.400 \mathrm{~s}$ & $e_{3}$ & -1.3250 \\
\hline
\end{tabular}

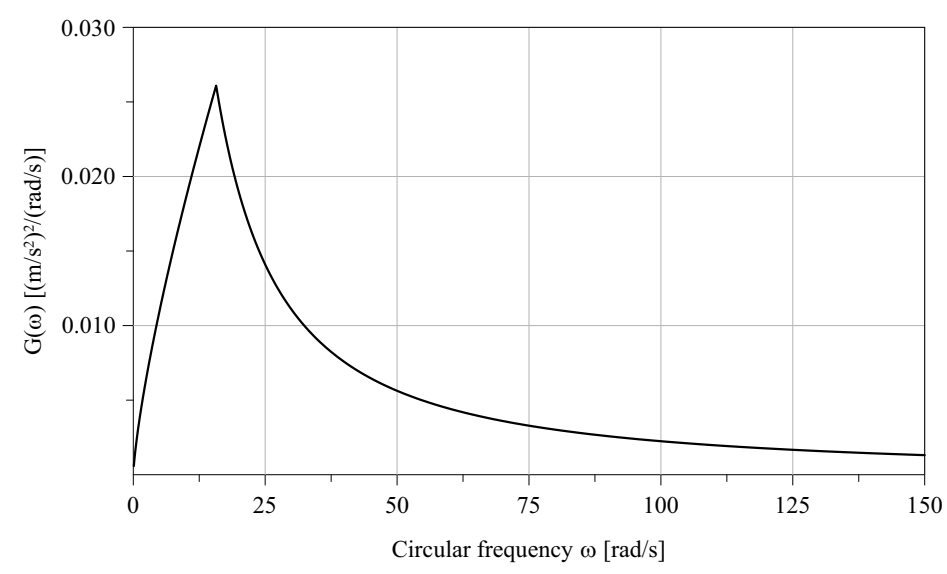

(a)

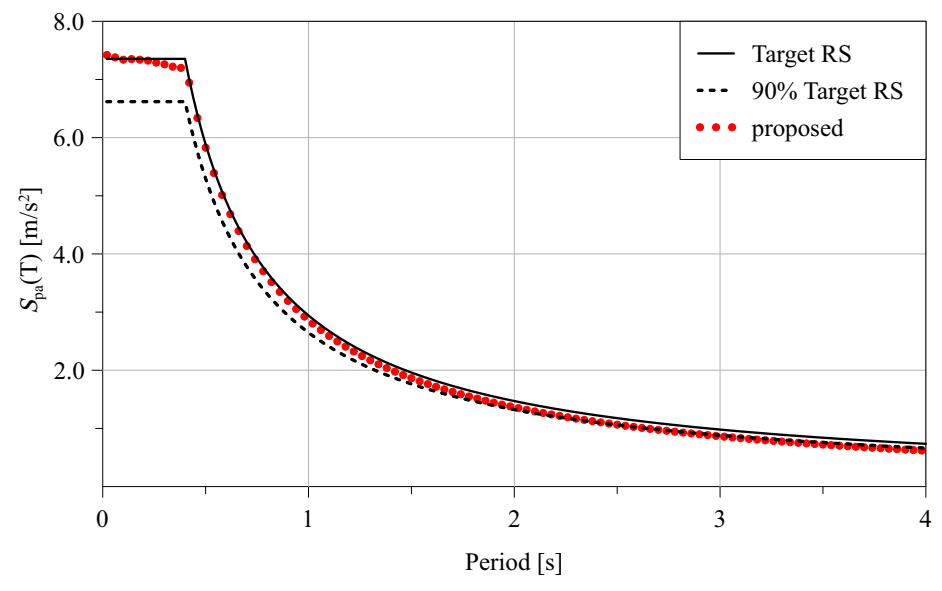

(b)

Figure 6: (a) PSD function consistent with the two-branch RS (parameters listed in Table 4; (b) Pseudo-acceleration RS: target (black solid line), $10 \%$ tolerance (black dashed line) and generated RS obtained with proposed approach (red dots).

292 et al. [52] has been adopted:

$$
\varphi_{\mathrm{JHT}}(t)= \begin{cases}\left(t / t_{1}\right)^{2}, & t<t_{1} \\ 1, & t_{1} \leq t \leq t_{2} \\ \exp \left(-\left(t-t_{2}\right)\right), & t>t_{2}\end{cases}
$$

293 where $t_{1}=5 \mathrm{~s}$ and $t_{2}=t_{1}+T_{\mathrm{S}}$. For each ground acceleration sample, the equation of motion of a linear SDOF system 294 with assigned period and damping ratio has been numerically integrated with the 4th-order Runge-Kutta method. 295 Finally, the relevant response statistics and the peak values of displacements, velocities and accelerations have been 296 computed in the pseudo-stationary range $\left[t_{1}, t_{2}\right]$.

\section{5. A case study: reliability analysis of a base isolated MDOF structure}

298 Based on the formulation developed on the previous sections, a very wide range of structural problems can be tackled. 299 In this section, for demonstration purposes, a base-isolated building taken from Ref. [42] is analyzed as case study. 300 This structure is a five-story base-isolated building with irregular distribution of mass and stiffness, both in plan and 301 in elevation. The damping ratio of the superstructure and isolated base are assumed to be $\zeta_{\mathrm{s}}=0.02$ and $\zeta_{\mathrm{b}}=0.12$, 302 respectively. Detailed information on the spatial distribution of mass and stiffness can be found in Ref. [42] and, as ${ }_{303}$ much as possible, a notation consistent with Ref. [42] has been adopted in what follows.

304 The case study has been selected due to two main reasons: $i$ ) first, the classical RS method may lead in this case 305 to an inaccurate evaluation of the dynamic response, mainly due to the empirical scaling of the spectral ordinates for ${ }_{306}$ different values of the viscous damping ratio; and $i$ ) second, the case study allows highlighting the computational 307 advantages of the proposed approach, in comparison with traditional numerical techniques. 


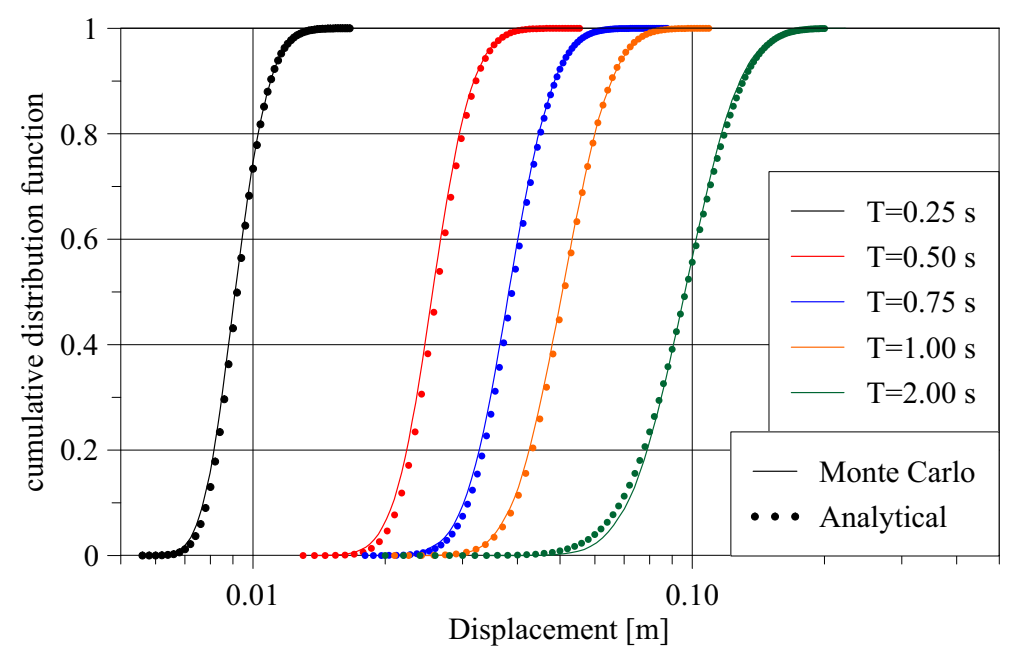

Figure 7: CDF of peak displacements of SDOF systems excited by seismic acceleration characterized by the PSD having parameters in Table 2. Analytic approach vs Monte Carlo simulations.

308 In the following, the governing equation of the seismic response are determined by using a sub-structure modelling 309 approach and a double transformation of coordinates, in order to reduce the computational burden. Then, the proposed 310 formulation is applied for the determination of the spectral moments required for the reliability analysis.

\section{5.1. Governing equations}

${ }_{312}$ Considering the isolated base and the superstructure as two dynamically interconnected systems, and assuming an 313 in-plan rigid behavior of the 5 stories in elevation, the building possesses $n=18$ degrees of freedom, i.e. three for

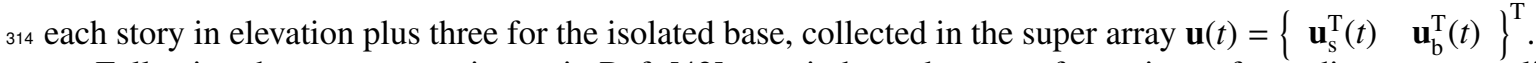

${ }_{315}$ Following the same reasoning as in Ref. [42], two independent transformations of coordinates are applied to the 316 superstructure and the base:

$$
\mathbf{u}_{\mathrm{s}}(t)=\boldsymbol{\Phi}_{\mathrm{s}} \cdot \mathbf{q}_{\mathrm{s}}(t) ; \quad \mathbf{u}_{\mathrm{b}}(t)=\boldsymbol{\Phi}_{\mathrm{b}} \cdot \mathbf{q}_{\mathrm{b}}(t),
$$

317 where $\boldsymbol{\Phi}_{\mathrm{s}}$ and $\boldsymbol{\Phi}_{\mathrm{b}}$ are the $\left((n-3) \times \ell_{\mathrm{s}}\right)$ and $(3 \times 3)$ real-valued modal matrices associated to superstructure and base, ${ }_{318}$ respectively, and $\mathbf{q}(t)=\left\{\mathbf{q}_{\mathrm{s}}^{\mathrm{T}}(t) \quad \mathbf{q}_{\mathrm{b}}^{\mathrm{T}}(t)\right\}^{\mathrm{T}}$ is an array collecting the $\ell=\ell_{\mathrm{s}}+3$ modal coordinates of the coupled ${ }_{319}$ dynamic system, $\ell_{\mathrm{S}}$ being the number of modes retained for the superstructure only.

${ }_{320}$ As the equations of motion are still coupled in the transformed vector space, a second transformation of coordi321 nates is applied:

$$
\mathbf{q}(t)=\psi\left(\alpha_{\mathrm{g}}\right) \cdot \mathbf{y}(t),
$$

322 where $\psi\left(\alpha_{\mathrm{g}}\right)$ is a convenient square matrix of size $\ell$, which depends on the epicentral direction of the seismic action, ${ }_{323} \alpha_{\mathrm{g}}$, and requires the solution of a third real-valued eigen-problem, this time of a reduced size $\ell$.

${ }_{324}$ By neglecting any coupling in terms of damping forces for the resulting generalized modal coordinates in the 325 vector $\mathbf{y}(t)$, the equations governing the seismic motion of this type of structures take the same form as Eq. (25), 326 in which $\mathbf{p}=\{1, \ldots, 1\}^{\mathrm{T}}$ is conveniently defined as a unit vector, while the modal transformation matrix $\mathbf{\Phi}$ can be 327 expressed as:

$$
\boldsymbol{\Phi}=\left[\begin{array}{cc}
\boldsymbol{\Phi}_{\mathrm{s}} & \mathbf{0} \\
\mathbf{0} & \boldsymbol{\Phi}_{\mathrm{b}}
\end{array}\right] \cdot \boldsymbol{\psi}\left(\alpha_{\mathrm{g}}\right) .
$$

${ }_{328}$ The matrices of spectral moments of order $m$ in the geometrical space are, hence, determined by applying the proce${ }_{329}$ dure outlined in the previous section, i.e. Eqs. (26) to (31).

330 It should be stressed here that the two-stage modal transformation for base-isolated building structures returns a ${ }_{331}$ dynamic model which is classically damped, i.e. the damping forces do not couple the generalized modal coordinates, 332 but the viscous damping ratio in the first three modes of vibration (associated with relatively large deformation in the ззз seismic isolators) tends to be much higher than that in the higher modes of vibration. 
Table 5: Modal participation masses for the analyzed base-isolated building.

\begin{tabular}{cccc}
\hline \multirow{2}{*}{ Mode } & Period [s] & \multicolumn{2}{c}{ Modal participation mass [\%] } \\
& & $\alpha_{\mathrm{g}}=0^{\circ}$ & $\alpha_{\mathrm{g}}=90^{\circ}$ \\
\hline $\mathbf{1}$ & 2.086 & 7.32 & $\mathbf{9 1 . 8 0}$ \\
$\mathbf{2}$ & 2.077 & $\mathbf{9 1 . 9 3}$ & 7.65 \\
3 & 1.806 & 0.73 & 0.50 \\
4 & 0.256 & 0.00 & 0.04 \\
5 & 0.240 & 0.01 & 0.00 \\
6 & 0.211 & 0.01 & 0.00 \\
\hline
\end{tabular}

${ }_{334}$ In the numerical application, the seismic analysis of the base-isolated building has been performed with reference 335 to the RS already defined in Table 2 and for two epicentral directions, namely $x$ and $y$, orthogonal to each other and ${ }_{336}$ parallel to the main structural frames in the superstructure (say, $\alpha_{\mathrm{g}}=0^{\circ}$ and $\alpha_{\mathrm{g}}=90^{\circ}$ ). The modal participation ${ }_{337}$ masses for the first six modes of vibration are reported in Table 5, considering both epicentral directions; then modes 338 associated with relatively large displacements of the isolated base are embolden.

\section{3з9 5.2. Reliability analysis}

${ }_{340}$ The statistical distributions of the peak values of the stories' horizontal displacements and rotations collected in the ${ }_{341}$ vector $\mathbf{u}(t)$ have been analytically determined with the approach presented in the previous sections. Results are first ${ }_{342}$ presented in Table 6 in terms of median peak values, where the degree of freedom $u_{e}^{(s)}$ stands for the horizontal ${ }_{343}$ displacement of the center of mass of the $s$ th story in the direction $e$ and $\theta_{z}^{(s)}$ is the rotation of the sth story about 344 the vertical axis. The results offered by the proposed procedure, based on the calculation of the analytical spectral 345 moments (ASM), are compared therein with the values obtained by the response spectrum method (RSM) using the ${ }_{346} \mathrm{CQC}$ (complete quadratic combination) of the modal responses. As benchmark solutions, Monte Carlo simulations 347 (MCS) have also been performed considering 20,000 samples with stationary observation time $T_{\mathrm{S}}=20 \mathrm{~s}$.

${ }_{348}$ The proposed analytical method provides results which are much closer to MCS than the RSMn does; while in 349 the first case the largest percentage errors are in the order of 17\% maximum, the RSM percentage can exceed $300 \%$. ${ }_{350}$ Furthermore, the computational effort required using the analytical formulae proposed in the previous sections is ${ }_{351}$ negligible when compared with the computational time needed for MCS.

$352 \quad$ Figures 8 and 9 plot the CDFs (cumulative distribution functions) of the peak horizontal displacements of the ${ }_{353}$ center of mass for all the stories of the building, including the isolated base, in both $x$ and $y$ direction and for both epi${ }_{354}$ central directions. The CDFs analytically determined with the proposed approach (ASM, dashed lines) are compared 355 against the numerical ones, obtained through MCS (solid lines). As shown, the maximum percentage discrepancy be${ }_{356}$ tween the two methods occurs for the displacement along the $y$ direction due to a seismic excitation in the $x$ direction. ${ }_{357}$ Furthermore, the percentage inaccuracies of the ASM are generally lower than those of the RSM (see Table 6). On 358 the other hand, since the proposed method only requires the use of closed-form expressions, the computational effort 359 required to determine the full statistical distribution of the peak response by ASM is negligible when compared to 360 both MCS. From a practical perspective, the complete knowledge of the maximum peak displacements can be used ${ }_{361}$ for structural assessment purposes (e.g. comparing actual base displacements with isolator capacities), meaning that 362 the rather significant improvement in computational efficiency makes the proposed method particular convenient for ${ }_{363}$ preliminary response analyses and parametric investigations requiring a large number of iterations.

\section{6. Conclusions}

${ }_{365}$ In this paper, closed-form expressions for the spectral moments of the stationary Gaussian response of linear struc366 tures excited by spectrum-consistent seismic excitations have been determined. In particular, spectral moments up to ${ }_{367}$ the third order have been determined for SDOF (single-degree-of-freedom) oscillators and up to the second order for ${ }_{368}$ MDOF (multi-degree-of-freedom) structures. Noticeably, these are the spectral moment needed to estimate the peak 


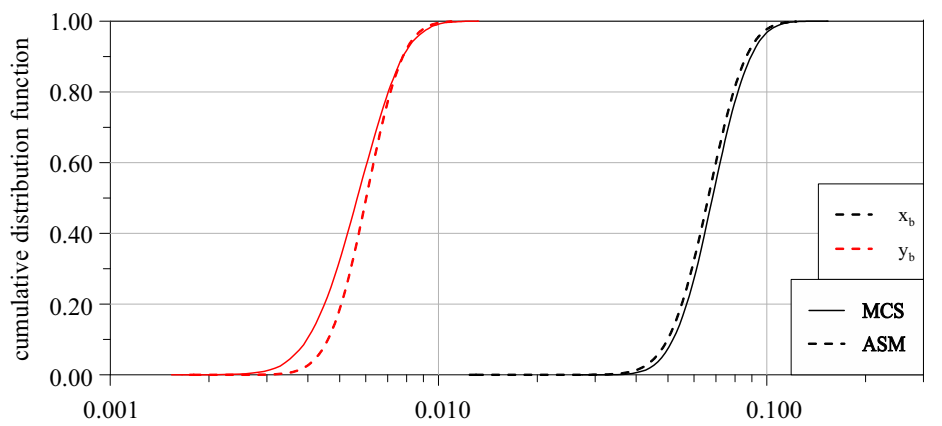

a)

Storey displacements [m]

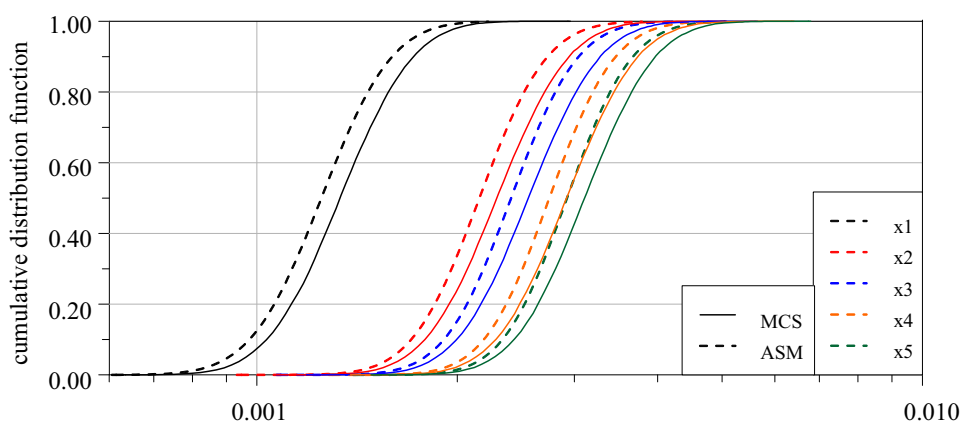

b)

Storey displacements [m]

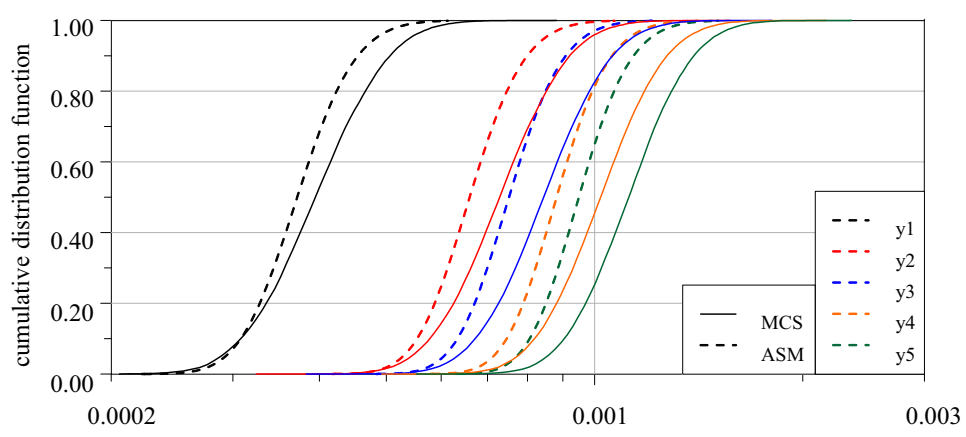

c)

Storey displacements [m]

Figure 8: CDF of the peak displacements of the base-isolated building for epicentral direction $x$; comparison between values by analytical spectral moments (ASM) and Monte Carlo simulations (MCS). 


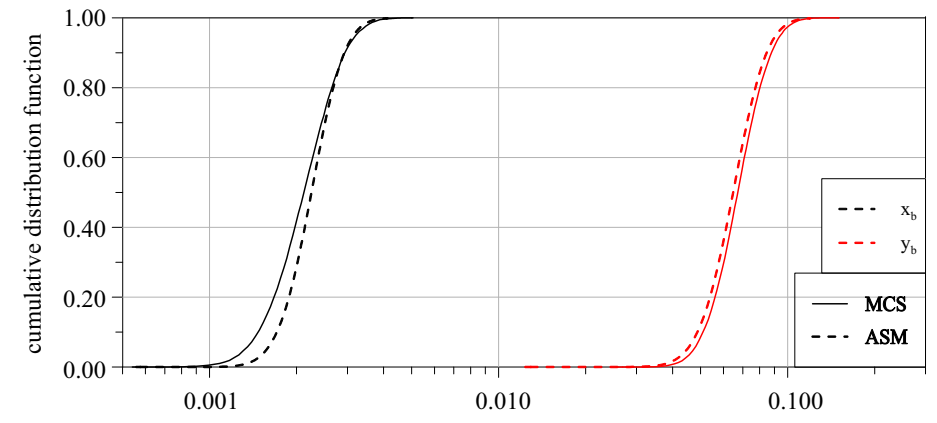

a)

Storey displacements [m]

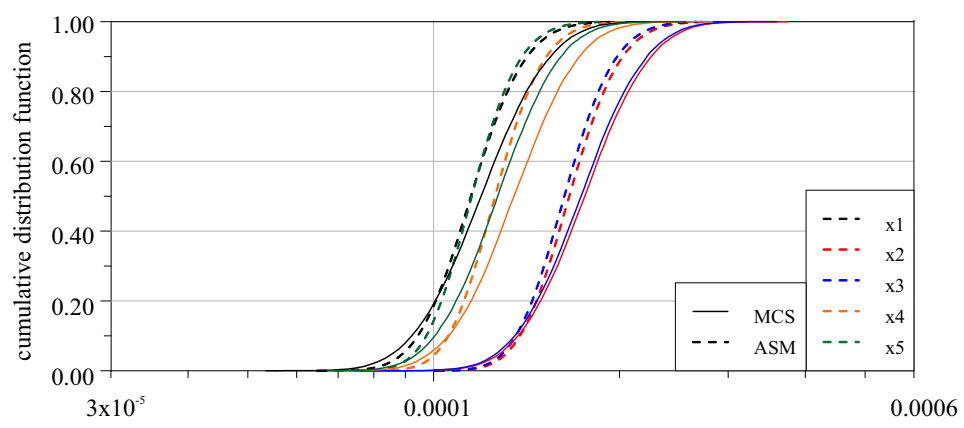

b)

Storey displacements [m]

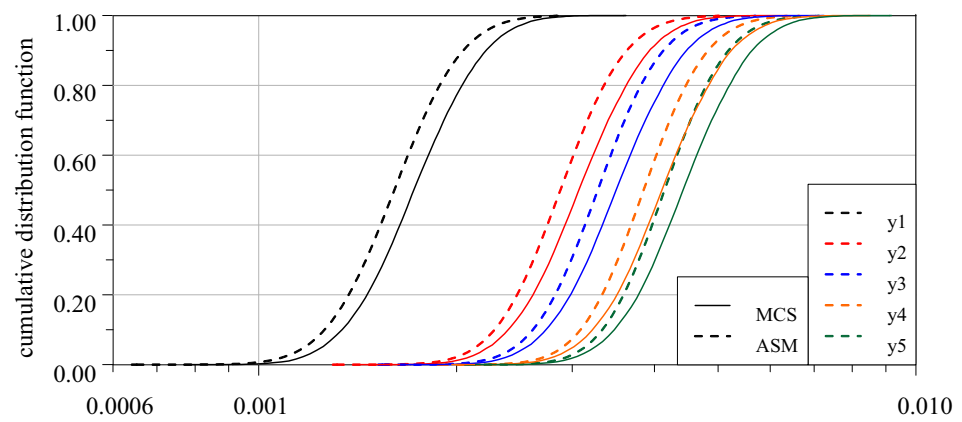

c)

Storey displacements [m]

Figure 9: CDF of the peak displacements of the base-isolated building for epicentral direction $y$; comparison between values by analytical spectral moments (ASM) and Monte Carlo simulations (MCS). 
Table 6: Mean peak displacements $[\mathrm{mm}]$ and rotations $\left[10^{-3} \mathrm{rad}\right]$ for two orthogonal epicentral directions, obtained by Monte Carlo simulations (MCS), response spectral method (RSM) and proposed approach (ASM).

\begin{tabular}{lcccccccccc}
\hline \multirow{2}{*}{ DOF } & \multicolumn{3}{c}{ Epicentral direction $x$} & \multicolumn{3}{c}{ Error [\%] } & \multicolumn{3}{c}{ Epicentral direction $y$} & \multicolumn{2}{c}{ Error [\%] } \\
& MCS & RSM & ASM & RSM & ASM & MCS & RSM & ASM & RSM & ASM \\
\hline$u_{1}=u_{x}^{(1)}$ & 1.37 & 1.40 & 1.25 & 2.1 & -9.2 & 0.12 & 0.29 & 0.11 & 136.6 & -6.7 \\
$u_{2}=u_{y}^{(1)}$ & 0.41 & 0.58 & 0.37 & 42.5 & -8.7 & 1.77 & 2.70 & 1.60 & 52.6 & -9.5 \\
$u_{3}=\theta_{z}^{(1)}$ & 0.04 & 0.05 & 0.04 & 34.2 & -8.9 & 0.03 & 0.07 & 0.03 & 120.5 & -4.8 \\
$u_{4}=u_{x}^{(2)}$ & 2.36 & 2.37 & 2.15 & 0.7 & -8.8 & 0.18 & 0.44 & 0.17 & 142.6 & -8.2 \\
$u_{5}=u_{y}^{(2)}$ & 0.75 & 1.00 & 0.66 & 34.2 & -12.0 & 3.15 & 4.74 & 2.87 & 50.4 & -9.1 \\
$u_{6}=\theta_{z}^{(2)}$ & 0.07 & 0.09 & 0.06 & 25.8 & -12.2 & 0.05 & 0.10 & 0.04 & 118.4 & -7.1 \\
$u_{7}=u_{x}^{(3)}$ & 2.63 & 2.64 & 2.40 & 0.3 & -8.7 & 0.18 & 0.45 & 0.16 & 154.1 & -8.6 \\
$u_{8}=u_{y}^{(3)}$ & 0.87 & 1.14 & 0.75 & 31.4 & -13.3 & 3.59 & 5.38 & 3.27 & 49.7 & -9.1 \\
$u_{9}=\theta_{z}^{(3)}$ & 0.08 & 0.10 & 0.07 & 23.6 & -13.2 & 0.05 & 0.10 & 0.04 & 123.7 & -7.9 \\
$u_{10}=u_{x}^{(4)}$ & 3.00 & 2.98 & 2.74 & -0.5 & -8.7 & 0.14 & 0.41 & 0.13 & 198.6 & -9.4 \\
$u_{11}=u_{y}^{(4)}$ & 1.04 & 1.32 & 0.88 & 26.2 & -15.8 & 4.22 & 6.26 & 3.84 & 48.4 & -9.0 \\
$u_{12}=\theta_{z}^{(4)}$ & 0.10 & 0.12 & 0.08 & 19.7 & -14.3 & 0.04 & 0.09 & 0.03 & 141.8 & -9.7 \\
$u_{13}=u_{x}^{(5)}$ & 3.19 & 3.16 & 2.91 & -1.0 & -8.7 & 0.13 & 0.40 & 0.12 & 207.8 & -11.6 \\
$u_{14}=u_{y}^{(5)}$ & 1.15 & 1.41 & 0.95 & 23.2 & -17.3 & 4.54 & 6.70 & 4.13 & 47.6 & -9.1 \\
$u_{15}=\theta_{z}^{(5)}$ & 0.11 & 0.12 & 0.09 & 17.2 & -15.3 & 0.03 & 0.08 & 0.03 & 141.9 & -11.4 \\
$u_{16}=u_{x}^{(b)}$ & 70.31 & 78.43 & 66.17 & 11.6 & -5.9 & 2.20 & 9.51 & 2.25 & 332.9 & 2.3 \\
$u_{17}=u_{y}^{(b)}$ & 5.86 & 14.16 & 6.01 & 141.6 & 2.5 & 69.13 & 115.61 & 64.68 & 67.2 & -6.4 \\
$u_{18}=\theta_{z}^{(b)}$ & 0.51 & 1.13 & 0.52 & 124.7 & 3.4 & 0.44 & 1.53 & 0.46 & 245.5 & 4.1 \\
\hline
\end{tabular}

369 response of both SDOF oscillators and MDOF structures using the classical Vanmarke's peak factor. While conven370 tionally numerical integrations are required for this type of analysis, the proposed formulation delivers closed-form 371 expressions for the probability distribution of the structural peak response.

372 Numerical validation of the proposed approach has been achieved by comparing the derived analytical expressions 373 with the results of MCS (Monte Carlo simulations), studying several cases of SDOF oscillators with varying natural 374 periods of vibration and viscous damping ratios, and considering also the case of a multi-story base-isolated building 375 taken from the literature.

376 Based on the results of the numerical examples, the following key conclusions of general significance can be 377 drawn:

- For the case of SDOF oscillators, excellent agreement has been found between the proposed closed-form expressions and the spectral moments calculated numerically. Only minor differences have been observed for relatively large natural periods of vibration (i.e. $T>2 \mathrm{~s}$ ) and large viscous damping ratios (i.e. $\zeta>0.10$ ), which are consistent with the assumptions underpinning the formulation itself.

- Using the analytical spectral moments to reproduce the target response spectrum returns excellent results. The difference between reproduced and target 4-branch response spectra (RS) are below the 10\% threshold prescribed by Eurocode 8 for acceptance. This has been verified also for RS proposed by other international building codes, including those with only 2 and 3 branches, always providing excellent results.

- For SDOF oscillators, very good agreement has also been found between the probability distributions of the peak displacements determined by MCS and those obtained with the proposed formulation. Similar to the case of the spectral moments, minor discrepancies have only been observed for structural periods over two seconds.

- The case study performed on a base-isolated multi-story building (with isolation period $T_{\text {iso }}=2.086 \mathrm{~s}$ ) has shown that, in comparison with MCS, the proposed approach entails negligible computational effort while achieving a level of accuracy that is either comparable or higher than the RSM (response spectrum method). 
- The computational efficiency of the proposed analysis method, relying on closed-form expressions, particularly shines when utilized for processes involving a large number of iterations, such as preliminary parametric analyses or seismic design optimization through, for example, the application of heuristic methods.

\section{${ }_{395}$ References}

[1] Clough R, Penzien J. Dynamics of Structures. Civil Engineering Series; McGraw-Hill; 1993. ISBN 9780071132411.

2] Lin Y, Cai G. Probabilistic Structural Dynamics: Advanced Theory and Applications. Engineering Reference Series; McGraw-Hill; 2004. ISBN 9780071438001.

399 [3] Ahmadi G. Generation of artificial time-histories compatible with given response spectra—a review. Solid Mechanics Archives 1979;4:207$400 \quad 39$.

401 [4] Kanai K. Semi-empirical formula for the seismic characteristics of the ground. Bulletin of the Earthquake Research Institute 1957;

402 [5] Tajimi H. A statistical method of determining the maximum response of a building structure during an earthquake. In: 2nd World Conference of Earthquake Engineering. Tokyo and Kyoto; 1960,

[6] Falsone GaNF. Stochastic modelling of earthquake excitation following the EC8: power spectrum and filtering equations. European Earthquake Engineering 2000;39(1):3-12.

6 [7] CEN . Eurocode 8: Design of structures for earthquake resistance - Part 1: General rules, seismic actions and rules for buildings, EN 1998-1. Brussels: Comité Européen de Normalisation; 2004.

408 [8] Pfaffinger DD. Calculation of Power Spectra from Response Spectra. Journal of Engineering Mechanics 1983;109(1):357-72. doi:10.1061/(ASCE)0733-9399(1983)109:1(357).

410 [9] Preumont A. The generation of spectrum compatible accelerograms for the design of nuclear power plants. Earthquake Engineering \& Structural Dynamics 1984;12(4):481-97. doi:10.1002/eqe.4290120405.

412 [10] Preumont A. A method for the generation of artificial earthquake accelerograms. Nuclear Engineering and Design 1980;59(2):357-68. 413 doi:10.1016/0029-5493(80)90205-8.

414 [11] Kaul MK. Stochastic characterization of earthquakes through their response spectrum. Earthquake Engineering \& Structural Dynamics 415 1978;6(5):497-509. doi:10.1002/eqe.4290060506.

416 [12] Sundararajan C. An iterative method for the generation of seismic power spectral density functions. Nuclear Engineering and Design $417 \quad$ 1980;61(1):13-23. doi:10.1016/0029-5493(80)90074-6.

418 [13] Unruh JF, Kana DD. An iterative procedure for the generation of consistent power/response spectrum. Nuclear Engineering and Design $419 \quad 1981 ; 66(3): 427-35$. doi:10.1016/0029-5493(81)90172-2.

420 [14] Cacciola P, Colajanni P, Muscolino G. Combination of Modal Responses Consistent with Seismic Input Representation. Journal of Structural $421 \quad$ Engineering 2004;130(1):47-55. doi:10.1061/(ASCE)0733-9445(2004)130:1(47).

422 [15] Crespi PG, Floris C, Paganini p. A Probabilistic Method for Generating Spectrum Compatible Earthquake Time Histories. European $423 \quad$ Earthquake Engineering 2002;16(3):3-17.

424 [16] Cacciola P. A stochastic approach for generating spectrum compatible fully nonstationary earthquakes. Computers and Structures $425 \quad$ 2010;88(15-16):889-901. doi:10.1016/j.compstruc.2010.04.009.

426 [17] Cacciola P, Zentner I. Generation of response-spectrum-compatible artificial earthquake accelerograms with random joint time-frequency 427 distributions. Probabilistic Engineering Mechanics 2012;28:52-8. doi:10.1016/j.probengmech.2011.08.004.

428 [18] Giaralis A, Spanos P. Wavelet-based response spectrum compatible synthesis of accelerograms-Eurocode application (EC8). Soil Dynamics $429 \quad$ and Earthquake Engineering 2009;29(1):219-35. doi:10.1016/j.soildyn.2007.12.002.

430 [19] Giaralis A, Spanos PD. Derivation of response spectrum compatible non-stationary stochastic processes relying on Monte Carlo-based peak 431 factor estimation. Earthquakes and Structures 2012;3(3_4):581-609.

432 [20] Conte JP, Peng BF. Fully Nonstationary Analytical Earthquake Ground-Motion Model. Journal of Engineering Mechanics 1997;123(1):15$433 \quad$ 24. doi:10.1061/(ASCE)0733-9399(1997)123:1(15).

434 [21] Gupta I, Trifunac M. Defining equivalent stationary PSDF to account for nonstationarity of earthquake ground motion. Soil Dynamics and $435 \quad$ Earthquake Engineering 1998;17(2):89-99. doi:10.1016/S0267-7261(97)00031-6.

436 [22] Spanos P, Giaralis A, Politis N. Time-frequency representation of earthquake accelerograms and inelastic structural response records using 437 the adaptive chirplet decomposition and empirical mode decomposition. Soil Dynamics and Earthquake Engineering 2007;27(7):675-89. $438 \quad$ doi:10.1016/j.soildyn.2006.11.007.

439 [23] Cecini D, Palmeri A. Spectrum-compatible accelerograms with harmonic wavelets. Computers \& Structures 2015;147:26-35. 440 doi:10.1016/j.compstruc.2014.10.013.

441 [24] Spanos PD, Giaralis A, Jie L. Synthesis of accelerograms compatible with the Chinese GB 50011-2001 design spectrum via harmonic 442 wavelets: Artificial and historic records. Earthquake Engineering and Engineering Vibration 2009;8(2):189-206. doi:10.1007/s11803-009$443 \quad 9017-4$

444 [25] Rezaeian S, Der Kiureghian A. A stochastic ground motion model with separable temporal and spectral nonstationarities. Earthquake $445 \quad$ Engineering \& Structural Dynamics 2008;37(13):1565-84. doi:10.1002/eqe.831.

446 [26] Priestley MB. Evolutionary spectra and non-stationary processes. Journal of the Royal Statistical Society Series B (Methodological) $447 \quad 1965 ; 27(2): 204-37$.

448 [27] Spanos PD. Non-stationary random vibration of a linear structure. International Journal of Solids and Structures 1978;14(10):861-7. 449 doi:10.1016/0020-7683(78)90076-8.

450 [28] Spanos PD, Vargas Loli LM. A statistical approach to generation of design spectrum compatible earthquake time histories. International 451 Journal of Soil Dynamics and Earthquake Engineering 1985;4(1):2-8. doi:10.1016/0261-7277(85)90029-4.

452 [29] Di Paola M, La Mendola L, Navarra G. Stochastic Seismic Analysis of Structures with Nonlinear Viscous Dampers. Journal of structural 453 engineering 2007;133(10):1475-8. doi:10.1002/stc.254. 
454 [30] Di Paola M, Navarra G. Stochastic seismic analysis of MDOF structures with nonlinear viscous dampers. Structural Control and Health $455 \quad$ Monitoring 2009;16(3):303-18. doi:10.1002/stc.254.

456 [31] Navarra G, Barone G, Lo Iacono F. Stochastic seismic analysis by using an analytical model of PSD consistent wit Response Spectra. Vienna $457 \quad$ Reccent Advance in Earthquake Engineering and Structural Dynamics (VEESD2013) 2013;2013(387):1-10.

458 [32] Barone G, Lo Iacono F, Navarra G, Palmeri A. A novel analytic model of power spectral density function coherent with earthquake re459 sponse spectra. In: M. Papadrakakis, V. Papadopoulos GS, editor. Proceedings of the 1st International Conference on Uncertainty Quan460 tification in Computational Sciences and Engineering (UNCECOMP 2015). Athens: Institute of Structural Analysis and Antiseismic Re-

461 search School of Civil Engineering National Technical University of Athens (NTUA) Greece. ISBN 978-960-99994-9-6; 2015, p. 394-406. 462 doi:10.7712/120215.4280.805.

463 [33] Vanmarcke EH. Properties of spectral moments with applications to random vibration. Journal of the Engineering Mehanics Division $464 \quad$ 1972;98(2):425-46.

465 [34] Vanmarcke EH. On the Distribution of the First-Passage Time for Normal Stationary Random Processes. Journal of Applied Mechanics $466 \quad 1975 ; 42(1): 215$. doi:10.1115/1.3423521.

467 [35] Vanmarcke EH. Chapter 8 - structural response to earthquakes. In: Lomnitz C, Rosenblueth E, editors. Seismic Risk and Engineering 468 Decisions; vol. 15 of Developments in Geotechnical Engineering. Elsevier; 1976, p. 287 -337. doi:https://doi.org/10.1016/B978-0-444$469 \quad$ 41494-6.50011-4.

470 [36] Miller KS, Rochwarger MM. On estimating spectral moments in the presence of colored noise. IEEE Transactions on Information Theory $471 \quad$ 1970;16(3):303-9. doi:10.1109/TIT.1970.1054460.

472 [37] Der Kiureghian A. Structural response to stationary excitation. Journal of the Engineering Mechanics Division ????;106(6):1195-213.

473 [38] Di Paola M, Muscolino G. Analytic evaluation of spectral moments. Journal of Sound and Vibration 1988;124(3):479-88. doi:10.1016/S0022$474 \quad 460 X(88) 81389-0$.

475 [39] Giaralis A, Spanos PD. Effective linear damping and stiffness coefficients of nonlinear systems for design spectrum based analysis. Soil $476 \quad$ Dynamics and Earthquake Engineering 2010;30(9):798-810. doi:10.1016/j.soildyn.2010.01.012.

477 [40] Spanos PD, Giaralis A. Third-order statistical linearization-based approach to derive equivalent linear properties of bilinear hysteretic systems 478 for seismic response spectrum analysis. Structural Safety 2013;44:59-69. doi:10.1016/j.strusafe.2012.12.001

479 [41] Mitseas IP, Kougioumtzoglou IA, Giaralis A, Beer M. A novel stochastic linearization framework for seismic demand estimation of hysteretic $480 \quad$ MDOF systems subject to linear response spectra. Structural Safety 2018;72:84-98. doi:10.1016/j.strusafe.2017.12.008.

481 [42] Muscolino G, Palmeri A, Versaci C. Damping-adjusted combination rule for the response spectrum analysis of base-isolated buildings. 482 Earthquake Engineering \& Structural Dynamics 2013;42(2):163-82. doi:10.1002/eqe.2200.

483 [43] Chopra A. Dynamics of Structures: Theory and Applications to Earthquake Engineering, 5th edition. Always learning; Pearson; 2017. ISBN $484 \quad 9780134555126$.

485 [44] Rosenblueth E. A basis for aseismic design of structures. Ph.d. thesis; University of Illinois at Urbana-Champaign; 1951

486 [45] Wilson EL, Der Kiureghian A, Bayo EP. A replacement for the SRSS method in seismic analysis. Earthquake Engineering \& Structural 487 Dynamics 1981;9(2):187-92. doi:10.1002/eqe.4290090207.

488 [46] International Code Council . International Building Code - IBC2012. USA; 2015.

489 [47] Ministerio de Vivienda . E.030 - Reglamento Nacional de Edificaciones. Diseño Sismo-Resistente (Technical Standard of Building, $490 \quad$ Earthquake-Resistant Design Chapter. Peru: Ministerio de Vivienda; 2006.

491 [48] Muscolino G, Palmeri A. Maximum response statistics of MDoF linear structures excited by non-stationary random processes. Computer 492 Methods in Applied Mechanics and Engineering 2005;194(12-16):1711-37. doi:10.1016/j.cma.2004.01.044.

493 [49] Muscolino G, Palmeri A. Peak response of non-linear oscillators under stationary white noise. Computers \& Structures 2007;85(5-6):255-63. doi:10.1016/j.compstruc.2006.10.005

495 [50] Muscolino G. Dinamica delle strutture con fondamenti ed applicazioni di ingegneria sismica e dinamica aleatoria. Bologna, Italy: Pitagora $496 \quad$ Editrice; 2012. ISBN 88-371-1858-9.

497 [51] Ministero delle Infrastrutture e dei trasporti . D.M. 17.01.2018 - Aggiornamento delle "Norme tecniche per le Costruzioni. Italy; 2018.

498 [52] Jennings P, Housner G, Tsai C. Simulated earthquake motions for design purpose. In: 4th World Conference on Earthquake Engineering; 499 vol. A-1. Santiago (Chile); 1969, p. 145-60.

\section{${ }_{500}$ Appendix A. Analytical derivation of PSD parameters}

${ }_{501}$ This section reports the analytical derivation of the PSD parameters defined in Eqs. (6) to (10). The procedure is ${ }_{502}$ initially presented in detail for the exponent $e_{1}$, and then a similar approach is applied in turn to derive the remaining ${ }_{503}$ parameters. In all cases, the starting point is Eq. (3), here reported for sake of clarity:

$$
G_{\ddot{U}_{\mathrm{g}}}(\omega)=\frac{\gamma}{\omega}\left[\left(\frac{S_{\mathrm{pa}}(\omega, \zeta)}{\eta_{U}(\omega, \zeta)}\right)^{2}-\int_{0}^{\omega} G_{\ddot{U}_{\mathrm{g}}}(\hat{\omega}) \mathrm{d} \hat{\omega}\right],
$$

\section{${ }_{504}$ Appendix A.1. Determination of exponent $e_{1}$}

${ }_{505}$ To determine the first parameter, the strategy is to particularize Eq. (A.1) considering two points belonging to the ${ }_{506}$ same branch of the RS-consistent PSD $\left(0 \leq \omega \leq \omega_{1}\right)$, and then algebraically manipulating the resulting mathematical 
507 relationships. It is worth reporting here, as a reminder, the mathematical expressions of both the RS and its consistent ${ }_{508}$ PSD functions (see Eq. (5)), both written in terms of the circular frequency $\omega=2 \pi / T$ :

$$
S_{\mathrm{pa}}(\omega)= \begin{cases}\alpha S_{0}\left(\frac{\omega_{1}}{\omega_{2}}\right)^{k_{1}}\left(\frac{\omega}{\omega_{1}}\right)^{k_{2},}, & 0 \leq \omega<\omega_{1} ; \\ \alpha S_{0}\left(\frac{\omega}{\omega_{2}}\right)^{k_{1}}, & \omega_{1} \leq \omega<\omega_{2} ; \\ \alpha S_{0}, & \omega_{2} \leq \omega \leq \omega_{3} ; \\ S_{0}\left[1+(\alpha-1) \frac{\omega_{3}}{\omega}\right], & \omega>\omega_{3} ;\end{cases}
$$

509

$$
G_{\ddot{U}_{\mathrm{g}}}(\omega)= \begin{cases}G_{0}\left(\frac{\omega_{1}}{\omega_{2}}\right)^{e_{2}}\left(\frac{\omega}{\omega_{1}}\right)^{e_{1}}, & 0 \leq \omega<\omega_{1} ; \\ G_{0}\left(\frac{\omega}{\omega_{2}}\right)^{e_{2}}, & \omega_{1} \leq \omega<\omega_{2} ; \\ G_{0}\left(\frac{\omega}{\omega_{2}}\right)^{e_{3}}, & \omega_{2} \leq \omega \leq \omega_{3} ; \\ G_{0}\left(\frac{\omega_{3}}{\omega_{2}}\right)^{e_{3}}\left(\frac{\omega}{\omega_{3}}\right)^{e_{4}}, & \omega>\omega_{3},\end{cases}
$$

Setting $\omega=\omega_{1}$ into Eq. (A.1) leads to:

$$
G_{0}\left(\frac{\omega_{1}}{\omega_{2}}\right)^{e_{2}}=\frac{\gamma}{\omega_{1}}\left[\frac{\left(\alpha S_{0}\left(\frac{\omega_{1}}{\omega_{2}}\right)^{k_{1}}\right)^{2}}{\eta_{U}^{2}\left(\omega_{1}, \zeta\right)}-\int_{0}^{\omega_{1}} G_{\ddot{U}_{g}}(\omega) \mathrm{d} \omega\right] .
$$

${ }_{511}$ Since the PSD function is given in analytical form, the integral term to the right-hand side can be solved in closed 512 form, leading to:

$$
G_{0} \omega_{2}\left(\frac{\omega_{1}}{\omega_{2}}\right)^{e_{2}+1} \frac{\gamma+e_{1}+1}{\gamma\left(e_{1}+1\right)}=\left(\frac{\alpha S_{0}}{\eta_{U}\left(\omega_{1}, \zeta\right)}\left(\frac{\omega_{1}}{\omega_{2}}\right)^{k_{1}}\right)^{2} .
$$

${ }_{513}$ Following the same two steps, but considering now the frequency $\omega=\omega_{1} / \rho$ (with $\rho>1$ ), the following expression is 514 obtained:

$$
G_{0} \omega_{2}\left(\frac{\omega_{1}}{\omega_{2}}\right)^{e_{2}+1} \frac{\gamma+e_{1}+1}{\gamma\left(e_{1}+1\right)}=\left(\frac{\alpha S_{0}}{\eta_{U}\left(\omega_{1} / \rho, \zeta\right)}\left(\frac{\omega_{1}}{\omega_{2}}\right)^{k_{1}}\right)^{2} \rho^{e_{1}+1-2 k_{2}} .
$$

${ }_{515}$ Comparison of equations (A.4) and (A.5) leads to:

$$
e_{1}=\frac{\ln \left(\frac{\eta_{U}^{2}\left(\omega_{1} / \rho, \zeta\right)}{\eta_{U}^{2}\left(\omega_{1}, \zeta\right)}\right)}{\ln (\rho)}+2 k_{2}-1
$$

516 In order to simplify the notation for the coming algebra, two new functions $A(\omega)$ and $B(\omega)$ are introduced as follows:

$$
A(\omega)=2 \eta_{U}(\omega) ;
$$

517

$$
B(\omega)=\exp \left[-q_{U}^{1.2}(\omega) \sqrt{\pi \ln (A(\omega))}\right],
$$


518 in which the explicit dependency on the viscous damping ratio $\zeta$ has been dropped to simplify the notation.

${ }_{519}$ The Vanmarcke's peak factor $\eta_{U}(\omega)$ can now be rewritten in the form:

$$
\eta_{U}(\omega)=\sqrt{2 \ln \{A(\omega)[1-B(\omega)]\}} .
$$

${ }_{520}$ Now, taking the limit of Eq. (A.6) with $\rho \rightarrow 1$, the following equation is obtained:

$$
e_{1}=2 k_{2}-1-L(\omega)
$$

521 where:

522

$$
L(\omega)=2 \frac{P(\omega)}{\eta_{U}^{2}(\omega)}
$$

$$
P(\omega)=1+\frac{B(\omega) \sqrt{\pi} q_{U}^{1.2}(\omega)}{2(1-B(\omega)) \sqrt{\ln (A(\omega))}} .
$$

${ }_{523}$ On the other hand, taking advantage of the positions in Eqs. (A.7), the first derivative of the peak factor $\eta_{U}(\omega)$ can be 524 written as:

$$
\frac{\mathrm{d}}{\mathrm{d} \omega} \eta_{U}(\omega)=\frac{1}{\omega} \frac{P(\omega)}{\eta_{U}(\omega)}
$$

${ }_{525}$ Comparison of Eqs. (A.10) and (A.11) gives then:

$$
L(\omega)=2 \omega \frac{\mathrm{d}}{\mathrm{d} \omega} \ln \left(\eta_{U}(\omega, \zeta)\right)
$$

526 confirming the validity of both eqs.(6) and (11).

${ }_{527}$ Appendix A.2. Determination of exponent $e_{2}$ and parameter $G_{0}$

${ }_{528}$ The closed-form expression for $e_{2}$ and $G_{0}$ are derived using a similar mathematical process, but considering in this ${ }_{529}$ case a new point positioned in the second branch of the PSD function, $\omega_{1} \leq \omega \leq \omega_{2}$. First, based on the analytical ${ }_{530}$ PSD model, the following integral can be solved in closed form:

$$
\int_{0}^{\omega_{2}} G_{\ddot{U}_{\mathrm{g}}}(\omega) \mathrm{d} \omega=G_{0} \omega_{2}\left[\frac{1}{1+e_{1}}\left(\frac{\omega_{1}}{\omega_{2}}\right)^{e_{2}+1}+\frac{1}{1+e_{2}}\left(1-\left(\frac{\omega_{1}}{\omega_{2}}\right)^{e_{2}+1}\right)\right] .
$$

${ }_{531}$ Then, rewriting Eq. (A.1) by setting $\omega=\omega_{2}$ and reordering all resulting terms, the following expression is obtained:

$$
\frac{G_{0} \omega_{2}}{\gamma}\left[\left(1-\left(\frac{\omega_{1}}{\omega_{2}}\right)^{e_{2}+1}\right) \frac{\gamma+e_{2}+1}{e_{2}+1}+\left(\frac{\omega_{1}}{\omega_{2}}\right)^{e_{2}+1} \frac{\gamma+e_{1}+1}{e_{1}+1}\right]=\left(\frac{\alpha S_{0}}{\eta_{U}\left(\omega_{2}\right)}\right)^{2} .
$$

${ }_{532}$ The latter returns $G_{0}$ as a function of the exponents $e_{1}$ and $e_{2}$, that can be rewritten in the form used for Eq. (10):

$$
G_{0}=\frac{\gamma}{\beta_{2} \omega_{2}}\left(\frac{\alpha S_{0}}{\eta_{U}\left(\omega_{2}\right)}\right)^{2}
$$

${ }_{533}$ where the coefficient $\beta_{2}$ has been given in Eq. (12).

${ }_{534}$ To derive the exponent $e_{2}$ in closed-form, Eq. (A.1) is rewritten for $\omega=\omega_{2} / \rho$ (assuming $1<\rho<\omega_{2} / \omega_{1}$ ):

$$
G_{0} \omega_{2}\left[\left(\frac{\omega_{1}}{\omega_{2}}\right)^{e_{2}+1}-\frac{1}{\rho^{e_{2}+1}}\right] \frac{\gamma+e_{2}+1}{\gamma\left(e_{2}+1\right)}=\left[\frac{\alpha S_{0}}{\eta_{U}\left(\omega_{1}\right)}\left(\frac{\omega_{1}}{\omega_{2}}\right)^{k_{1}}\right]^{2}-\left[\frac{\alpha S_{0}}{\eta_{U}\left(\omega_{2} / r h o\right)}\right]^{2} \frac{1}{\rho^{2 k_{1}}} .
$$

${ }_{535}$ By using Eq. (A.14) and Eq. (10), and simplifying opportunely, the following relationship is obtained:

$$
e_{2}+1=-\frac{1}{\ln (\rho)} \ln \left\{1+\beta_{2} \frac{e_{2}+1}{\gamma+e_{2}+1}\left[\left(\frac{\eta_{U}\left(\omega_{2}\right)}{\rho^{k_{1}} \eta_{U}\left(\omega_{2} / \rho\right)}\right)^{2}-1\right]\right\} \text {. }
$$


${ }_{536}$ The limit for $\rho \rightarrow 1$ of Eq. (A.17) leads to:

$$
e_{2}+1=-\frac{e_{2}+1}{\gamma+e_{2}+1} \beta_{2}\left(L\left(\omega_{2}\right)-2 k_{1}\right) .
$$

${ }_{537}$ Finally, by approximating that:

$$
\beta_{2} \cong \frac{\gamma+e_{2}+1}{e_{2}+1}
$$

${ }_{538}$ the closed-form expression for $e_{2}$ reported in Eq. (7) is obtained.

539 Appendix A.3. Determination of the exponent $e_{3}$

${ }_{540}$ For the evaluation of the exponent $e_{3}$, a point of the third branch is considered. Eq. (A.1) is then rewritten at the ${ }_{541}$ frequency $\omega=\rho \omega_{2}$ (again, with $\rho>1$ ):

$$
G_{0} \omega_{2}\left(\rho^{e_{3}+1}-1\right) \frac{\gamma+e_{3}+1}{\gamma\left(e_{3}+1\right)}=\left(\frac{\alpha S_{0}}{\eta_{U}\left(\rho \omega_{2}\right)}\right)^{2}-\left(\frac{\alpha S_{0}}{\eta_{U}\left(\omega_{2}\right)}\right)^{2} .
$$

${ }_{542}$ Substitution of Eq. (10) into Eq. (A.20) leads to:

$$
e_{3}+1=\frac{1}{\ln (\rho)} \ln \left\{1+\beta_{2} \frac{e_{3}+1}{\gamma+e_{3}+1}\left[\left(\frac{\eta_{U}\left(\omega_{2}\right)}{\eta_{U}\left(\rho \omega_{2}\right)}\right)^{2}-1\right]\right\} .
$$

${ }_{543}$ Performing the limit for $\rho \rightarrow 1$, as done in the previous subsection, the closed-form expression for the exponent $e_{3}$ is 544 obtained, as reported in Eq. (8).

${ }_{545}$ Appendix A.4. Determination of the exponent $e_{4}$

${ }_{546}$ The determination of the closed-form expression for the last exponent follows the same reasoning presented for the ${ }_{547}$ previous cases, considering Eq. (A.1) for two frequencies $\omega=\omega_{3}$ and $\omega=\rho \omega_{3}$ (again, with $\rho>1$ ), that is positioned ${ }_{548}$ on the last branch of the PSD function. The algebra, slightly more cumbersome than in the previous cases, leading to 549 the expression:

$$
e_{4}+1=\frac{1}{\ln (\rho)} \ln \left\{1+\beta_{3} \frac{e_{4}+1}{\gamma+e_{4}+1}\left[\left(\frac{\rho+\alpha+1}{\rho \alpha}\right)^{2}\left(\frac{\eta_{U}\left(\omega_{3}\right)}{\eta_{U}\left(\rho \omega_{3}\right)}\right)^{2}-1\right]\right\},
$$

${ }_{550}$ where $\beta_{3}$ has the expression reported in Eq. (13). Finally, by considering the limit $\rho \rightarrow 1$ and simplifying opportunely, ${ }_{551}$ the closed-form expression for the exponent $e_{4}$, reported in Eq. (9) is obtained. 